\title{
ANÁLISE DE PERIFIS PARA EXPERIMENTOS EM BLOCOS ALEATORIZADOS
}

\author{
Cristina Higashi
}

\author{
DISSERTAÇÃO DEFENDIDA \\ NO \\ INSTITUTO DE MATEMÁTICA E ESTATÍ́STICA \\ DA \\ UNIVERSIDADE DE SÃO PAULO \\ PARA OBTENÇÃO DO GRAU DE MESTRE \\ EM \\ ESTATÍSTICA
}

Área de concentração: Estatística

Orientador: Prof. Dr. Julio da Motta Singer

- São Paulo, abril de 1995 - 


\section{ANÁLISE DE PERFIS PARA EXPERIMENTOS EM BLOCOS ALEATORIZADOS}

Este exemplar corresponde à redação final da dissertação devidamente corrigida e defendida por Cristina Higashi e aprovada pela comissão julgadora

São Paulo, 26 de maio de 1995.

\section{Banca Examinadora}

Profa. Dra. Clarice Demétrio

Prof Dr. Dalton Francisco de Andrade

Prof Dr. Julio da Motta Singer 
Aos meus pais 


\section{AGRADECIMENTOS}

Durante a elaboração desta dissertação, contei com o inestimável apoio de várias pessoas amigas; sou-lhes muito grata pelo interesse contínuo e por todas as palavras de incentivo. Não poderia, entretanto, deixar de agradecer em especial

ao Professor Julio da Motta Singer, cuja função densidade de probabilidade se concentra em excelência, por sua dedicação, paciência e constante disposição a esclarecer quaisquer dúvidas.

ao Prof. Dalton Francisco de Andrade, que acompanhou o desenvolvimento da tese e enriqueceu-a com valiosas sugestões.

aos meus pais, à minha irmã Luciane e ao Damásio, por toda a força que me deram. 


\section{SUMÁRIO}

Resumo $\quad$ i

Abstract $\quad$ ii

1 Estudos Longitudinais 1

1.1 Introdução 1

1.2 Análise de Perfis 4

2 Análise de Perfis para Experimentos Completamente Aleatorizados 12

$\begin{array}{lll}2.1 & \text { Introdução } & 12\end{array}$

2.2 Medidas Resumo 14

2.3 Modelo Multivariado 16

2.4 Modelo Misto Univariado (tipo "SPLIT-PLOT") 18

2.5 Outros Modelos $\quad 22$

2.5.1 Modelo com Estrutura de Antedependência 22

2.5.2 Modelo com Estrutura Autoregressiva 23

2.5.3 Modelo de Efeitos Aleatórios 25

3 Análise de Perfis para Experimentos em Blocos Aleatorizados 27

3.1 Introdução $\quad 27$

3.2 Modelos de Efeitos Fixos para Blocos 28

3.3 Modelos de Efeitos Aleatórios para Blocos 32

4 Exemplo Numérico $\quad 35$

4.1 Análise dos Dados do Exemplo 1.1 35

4.2 Considerações Finais 46

$\begin{array}{ll}\text { Apêndice A } & 47\end{array}$

$\begin{array}{ll}\text { Apêndice B } & 49\end{array}$

Referências Bibliográficas $\quad 55$ 


\section{RESUMO}

$\mathrm{Na}$ primeira parte desta tese consideramos os uso de métodos padrão uni e multivariados para a Análise de Perfis em experimentos completamente aleatorizados. Descrevemos também brevemente outras estruturas de covariâncias usuais. Na segunda parte, introduzimos Blocos na análise e dam רs algumas sugestões de como adaptar os testes para experimentos completamente aleatorizados para o caso de experimentos em blocos completamente aleatorizados. 


\begin{abstract}
In the first part of this thesis we consider the use of standard uni and multivariate methods for Profile Analysis of completely randomized experiments. We also describe briefly other commonly ocurring covariance structures. In the second part, we introduce Blocks in the analysis and give some sugestions of how to adapt the standard tests for completely ramdomized experiments to the case of randomized block experiments.
\end{abstract}




\section{Capítulo 1}

\section{Estudos Longitudinais}

\subsection{Introdução}

Sob a denominação de Estudos Longitudinais consideram-se investigações científicas cujo interesse é voltado para a avaliação do comportamento de uma ou mais características ao longo de um determinado número de ocasiões ou de qualquer outra escala ordenada (distância de uma certa origem ou dosagem de uma determinada substância, por exemplo). Para facilitar a leitura, designaremos por "Tempo" essa escala ordenada. Estudos desta natureza são empregados em Agropecuária (Rowell and Walters (1976) ou Singer e Domenech (1989), por exemplo), em Economia (Dielman, (1983)), Ciências Sociais (Nesselroade and Baltes (1979)), Epidemiologia (Goldstein (1979)) e em pesquisas de Mercado de Trabalho (Heckman and Singer (1985)), entre outros. Os dados, em geral, consistem de um vetor de observações (perfil de resposta) para cada unidade de investigação (animal, pessoa, etc). As unidades de investigação podem estar classificadas em diferentes tratamentos segundo um ou mais fatores, tais como raça, procedência ou tipo de medicamento recebido.

Dentro da classe de Estudos Longitudinais convém destacar três categorias:

(a) Estudos baseados em planejamentos transversais ("cross-sectional"), onde se planeja observar cada unidade de investigação uma única vez;

(b) Estudos baseados em planejamentos longitudinais, onde se pretende observar cada unidade de investigação em todas as ocasiões para as quais o estudo foi globalmente dimensionado; 
(c) Estudos baseados em planejamentos mistos ("linked cross-sectional"), onde se pretende observar diferentes unidades de investigação em diferentes subconjuntos das ocasiões para as quais o estudo foi globalmente dimensionado.

Neste trabalho, estamos interessados em planejamentos longitudinais. Embora a obtenção de dados através desse tipo de planejamento exija esforços no acompanhamento das unidades de investigação durante a realização do estudo (ver Cook and Ware (1983), por exemplo), eles oferecem os seguintes atrativos:

- requerem menos unidades de investigação do que estudos semelhantes baseados em planejamentos transversais ou mistos;

- proporcionam um controle sobre diferenças entre as unidades de investigação (a variabilidade devido a diferenças entre as unidades é eliminada do erro experimental apropriado para comparações relativas a Tempo);

- permitem o estudo da evolução das respostas individuais ao longo do Tempo.

Uma discussão mais detalhada sobre a eficiência de planejamentos longitudinais em relação a planejamentos transversais pode ser encontrada em Cook and Ware (1983) ou em Domenech (1989, cap. 1), por exemplo.

Suponhamos um conjunto de $n$ unidades experimentais onde em cada unidade é observada uma variável resposta ao longo de $p$ condições conhecidas. Seja $y_{i}=\left(y_{i 1}, \ldots y_{i p}\right)^{t}$ 0 vetor de respostas associado à $i$-ésima unidade experimental. Um modelo linear para $y_{i}$ é dado por

$$
y_{i}=X_{i} \beta+\varepsilon_{i}
$$

onde $X_{i}(p \times q)$ é a matriz de especificação do modelo para o $i$-ésimo indivíduo, $\beta$ é um vetor $(q \times l)$ de parâmetros de regressão desconhecidos e os $\varepsilon_{i}$ 's são erros independentes com distribuição $\operatorname{Normal}\left(0, \Sigma_{i}\right)$, ou seja:

$$
y_{i} \sim \mathrm{N}\left(X_{i} \beta, \quad \Sigma_{i}\right)
$$

para $i=1, \ldots, n$ e $y_{1}, \ldots, y_{n}$ são independentes. Quando cada indivíduo é observado nas mesmas $p$ condições e não há observações incompletas, dizemos que o planejamento é balanceado em relação ao Tempo. Quando $X_{i}$ é independente de $i$, dizemos que 0 planejamento é completamente balanceado. O leitor interessado em análise de dados não balanceados no Tempo pode consultar Jennrich and Schluchter (1986), Andreoni (1989) ou Papescu (1995), por exemplo, para maiores detalhes. 
Dado que várias observações são feitas seqüencialmente na mesma unidade amostral, podem existir correlações não nulas entre elas. Ware (1985) constata que, tipicamente, as observações de uma mesma unidade amostral apresentam correlação serial positiva. Assim, embora nosso interesse esteja voltado para o vetor de médias, a matriz de covariâncias tem também um papel fundamental na análise, pois contém informações sobre possíveis correlações entre observarões realizadas na mesma unidade de investigação. Essa matriz tem influência direta nas estimativas da variabilidade dos parâmetros associados às médias e, em alguns casos, pode até afetar as próprias estimativas desses parâmetros. Uma estratégia de análise de dados com planejamento longitudinal consiste essencialmente na redução do número de parâmetros envolvidos (médias e covariâncias associadas à distribuição das observações) através da imposição de modelos parcimoniosos que relacionam sua variação com os tratamentos e com as condições de avaliação respeitando as estruturas naturais geradas pelo problema. Embora existam diferentes propostas para representar o comportamento médio da variável resposta, restringir-nos-emos aos modelos lineares que são de fácil interpretação e que, em geral, permitem ajustes satisfatórios. Com relação à matriz de covariâncias, vários modelos serão discutidos.

As técnicas clássicas de análise de dados longitudinais são direcionadas para 0 caso de dados balanceados em relação ao Tempo. Entre elas destacamos:

- Análise de Perfis;

- Análise de Curvas de Crescimento.

O principal objetivo da técnica de Análise de Perfis é testar hipóteses sobre os valores médios da variável resposta nas ocasiões de observação e compará-los entre os diferentes grupos de unidades experimentais. Na Análise de Curvas de Crescimento procuram-se ajustar curvas aos perfis médios de resposta, o que pode ser encarado como uma complementação da Análise de Perfís.

O interesse neste trabalho está voltado para a Análise de Perfis; o leitor interessado em Curvas de Crescimento poderá obter mais informações em Singer (1977), por exemplo. 


\subsection{Análise de Perfis}

A seleção de uma estratégia apropriada para a Análise de Perfis deve levar em conta os objetivos do estudo, a natureza do fenômeno em consideração, as suposições dos modelos e as características dos dados experimentais. Mais especificamente, a Análise de Perfis visa responder a três perguntas:

(a) Os perfis médios de resposta dos diferentes tratamentos são paralelos? (i.e., não existe interação entre Tratamento e Tempo?);

(b) se os perfis são paralelos, eles são coincidentes? (i.e., não existe efeito de Tratamento?);

(c) se os perfis são paralelos, eles são horizontais? (i.e., não existe efeito de Tempo?).

Métodos estatísticos para Análise de Perfis têm sido bastante difundidos por autores como Greenhouse and Geisser (1959), Winer (1971, caps. 4 e 6), Timm (1980), Jennrich and Schluchter (1986), etc., e têm sido implementados em alguns pacotes estatísticos, tais como BMDP, SAS e SPSS. Duas abordagens clássicas têm sido consideradas; a primeira é baseada em um modelo multivariado, onde nenhuma restrição é imposta a $\Sigma_{i}$ (ver, por exemplo: Timm (1980) ou Andrade e Singer (1986, cap. 2)) e a segunda baseia-se em um modelo misto univariado (tipo "SPLIT-PLOT"), em que as observações da mesma unidade de investigação têm variâncias iguais e correlações iguais (ver Winer (1971, cap.7) ou Andrade e Singer (1986, cap.2)). Mais recentemente, alternativas de análise baseadas em modelos intermediários e, muitas vezes, mais realistas, para a estrutura de covariância têm recebido maior atenção por parte de muitos autores; em particular citamos os modelos de efeitos aleatórios (ver Laird and Ware (1982) ou Andreoni (1989), por exemplo) e os modelos usualmente empregados na análise de Séries Temporais (ver LaVange and Helms (1983), por exemplo).

As técnicas usuais para a Análise de Perfis são, em sua maioria, dirigidas para o caso de experimentos onde as unidades de investigação são aleatoriamente atribuídas aos tratamentos. Em muitas situações, as unidades amostrais precisam ser divididas em blocos dentro dos quais a variabilidade é menor do que dentro do conjunto todo. Nesse caso, comparações experimentais podem freqüentemente ser feitas de modo muito mais eficiente do que se a existência de blocos fosse ignorada. 
Exemplo 1.1 (Singer e Domenech (1989)) Em um experimento realizado pelo Centro Nacional de Pesquisa de Suínos e Aves da EMBRAPA para se avaliar o efeito do nível de proteína em rações para leitões e o de sua suplementação com aminoácidos, foram utilizados 20 leitões, divididos em 4 baias, com base nos pesos iniciais (cada baia alojando 5 leitões), de modo a obter grupos os mais homogêneos possíveis. Os leitões dentro de cada baia foram designados, ao acaso, a um dos seguintes tratamentos, caracterizados pelos níveis de proteína e presença de aminoácidos:

(1) $20 \%$ de Proteína Bruta;

(2) $18 \%$ de Proteína Bruta;

(3) $16 \%$ de Proteína Bruta;

(4) $16 \%$ de Proteína Bruta + L-Lisina;

(5) $14 \%$ de Proteína Bruta + L-Treonina.

O crescimento dos animais foi avaliado através de pesagens, que foram realizadas no início do experimento e 7, 14 e 18 dias após o mesmo. Neste caso, baia é o fator Blocos com 4 níveis, os leitões são as unidades de investigação e as ocasiões de observação correspondem aos dias em que foram feitas as pesagens. Os dados encontram-se na Tabela 1.1.1. 
Tabela 1.1.1: Pesos (em kg) de leitões submetidos a diferentes dietas.

\begin{tabular}{cccccc}
\hline Bloco & Trat & Inicial & 7 dias & 14 dias & 18 dias \\
\hline 1 & 1 & 10,750 & 16,075 & 20,275 & 23,225 \\
1 & 2 & 10,875 & 14,950 & 18,425 & 22,300 \\
1 & 3 & 10,825 & 1 r 275 & 19,475 & 22,325 \\
1 & 4 & 10,875 & 15,850 & 20,250 & 23,025 \\
1 & 5 & 10,850 & 15,300 & 19,575 & 22,975 \\
2 & 1 & 10,425 & 15,050 & 19,550 & 22,650 \\
2 & 2 & 10,450 & 15,650 & 19,725 & 22,575 \\
2 & 3 & 10,375 & 13,325 & 16,775 & 18,825 \\
2 & 4 & 10,450 & 14,000 & 17,700 & 20,350 \\
2 & 5 & 10,450 & 15,400 & 19,600 & 22,375 \\
3 & 1 & 9,825 & 15,050 & 19,300 & 21,850 \\
3 & 2 & 9,850 & 14,700 & 18,475 & 22,150 \\
3 & 3 & 9,725 & 13,575 & 16,850 & 19,725 \\
3 & 4 & 9,800 & 14,050 & 18,275 & 20,125 \\
3 & 5 & 9,850 & 14,100 & 17,425 & 20,325 \\
4 & 1 & 9,275 & 13,775 & 17,525 & 20,375 \\
4 & 2 & 9,250 & 13,450 & 17,425 & 19,850 \\
4 & 3 & 9,350 & 13,025 & 16,750 & 19,075 \\
4 & 4 & 9,250 & 13,625 & 17,200 & 20,025 \\
4 & 5 & 9,350 & 13,325 & 16,675 & 19,200 \\
\hline & & & & &
\end{tabular}

Na Figura 1.1.1 encontram-se os perfis médios de resposta para cada tratamento. 
Figura 1.1.1: Perfis médios de resposta dos tratamentos

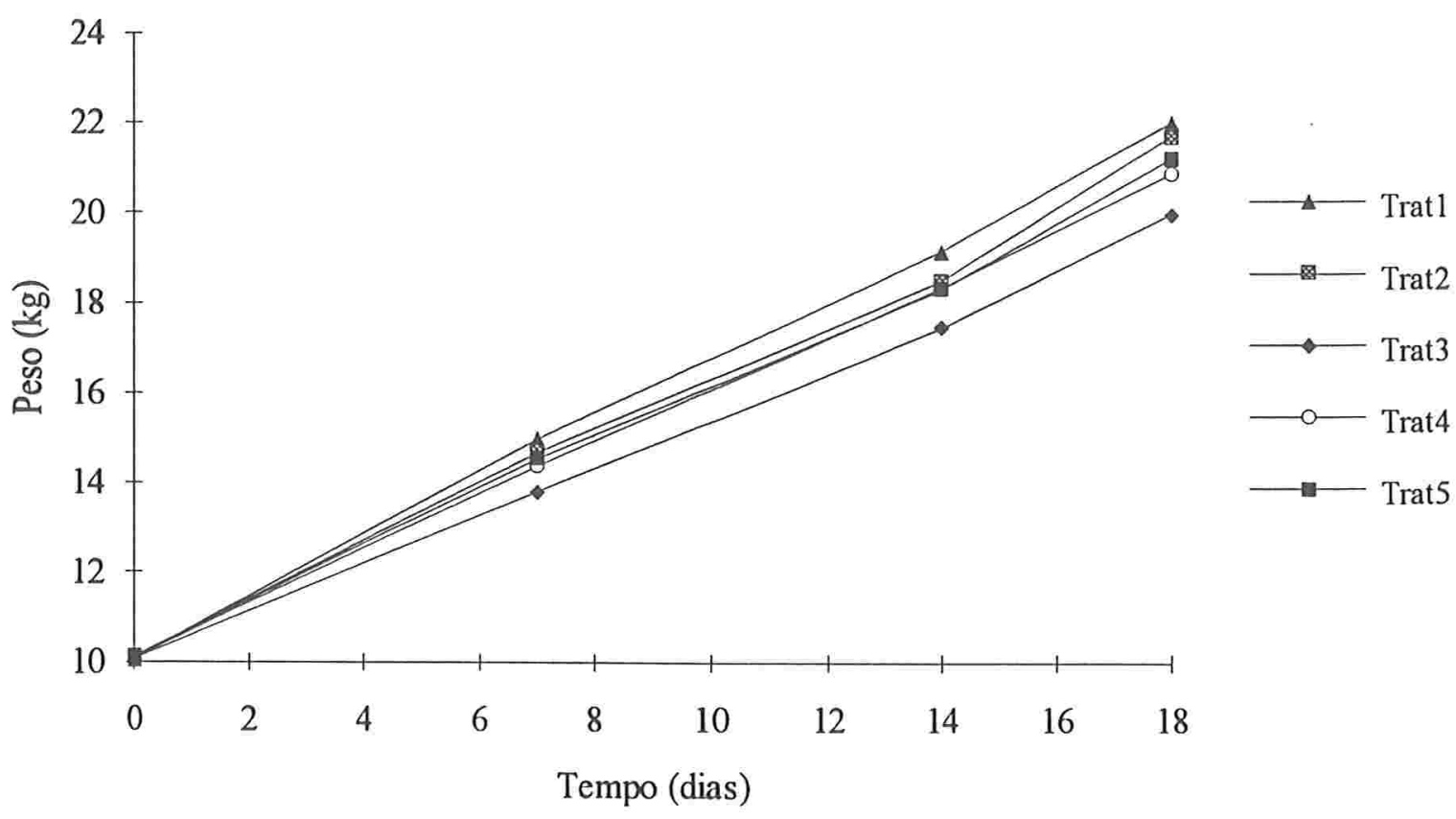

Exemplo 1.2 (Hoffman et al. (1985)). Em um experimento destinado a estudar os efeitos da ingestão de chumbo no crescimento de filhotes de falcão (American Kestrel), a variável resposta foi o peso dos filhotes, medido diariamente durante o período de aplicação dos tratamentos. 40 filhotes, 4 de cada um dos ninhos selecionados para 0 estudo, receberam doses orais diárias de um dos seguintes tratamentos durante seus primeiros 10 dias de vida:

(C) $5 \mu \mathrm{l}$ de óleo de milho (Grupo Controle);

(1) $25 \mathrm{mg} / \mathrm{kg}$ de pó de chumbo metálico diluído em óleo de milho;

(2) $125 \mathrm{mg} / \mathrm{kg}$ de pó de chumbo metálico diluído em óleo de milho;

(3) $625 \mathrm{mg} / \mathrm{kg}$ de pó de chumbo metálico diluído em óleo de milho.

Quatro, dos dez filhotes que receberam o Tratamento 3, morreram antes do término do experimento. Neste caso, $\underline{\text { ninho }}$ é o fator Blocos com 10 níveis (introduzido para reduzir um possível efeito de ninhadas), os filhotes de falcão são as unidades de investigação e as ocasiões de observação correspondem aos 10 dias de avaliação do estudo. Os dados encontram-se na Tabela 1.1.2. 
Tabela 1.1.2: Pesos (em g) de filhotes de falcão submetidos a dietas com diferentes concentrações de chumbo

\begin{tabular}{|c|c|c|c|c|c|c|c|c|c|c|c|}
\hline Bloco & Trat & dia 1 & dia 2 & dia 3 & $\operatorname{dia} 4$ & dia 5 & $\operatorname{dia} 6$ & dia 7 & dia 8 & dia 9 & dia 10 \\
\hline 1 & $\mathrm{C}$ & 12 & 15 & 21 & 26 & 29 & 35 & 56 & 62 & 76 & 81 \\
\hline 1 & 1 & 13 & 14 & 20 & 25 & 25 & 29 & 44 & 48 & 62 & 65 \\
\hline 1 & 2 & 13 & 15 & 20 & 24 & 25 & 30 & 37 & 43 & 52 & 55 \\
\hline 1 & 3 & 11 & 13 & 17 & 19 & 19 & 15 & - & - & - & - \\
\hline 2 & C & 16 & 19 & 26 & 33 & 40 & 49 & 63 & 72 & 86 & 90 \\
\hline 2 & 1 & 13 & 15 & 21 & 26 & 34 & 44 & 54 & 61 & 76 & 86 \\
\hline 2 & 2 & 15 & 17 & 22 & 28 & 34 & 40 & 50 & 58 & 74 & 82 \\
\hline 2 & 3 & 16 & 16 & 22 & 25 & 29 & 32 & 34 & 36 & 38 & 43 \\
\hline 3 & C & 10 & 14 & 19 & 25 & 32 & 44 & 52 & 68 & 77 & 82 \\
\hline 3 & 1 & 11 & 13 & 19 & 23 & 29 & 37 & 41 & 56 & 61 & 68 \\
\hline 3 & 2 & 11 & 13 & 18 & 22 & 29 & 37 & 41 & 53 & 62 & 66 \\
\hline 3 & 3 & 11 & 13 & 18 & 21 & 25 & 32 & 37 & 41 & 42 & 43 \\
\hline 4 & $\mathrm{C}$ & 12 & 16 & 19 & 24 & 25 & 41 & 46 & 65 & 69 & 75 \\
\hline 4 & 1 & 12 & 17 & 21 & 27 & 32 & 46 & 52 & 69 & 71 & 80 \\
\hline 4 & 2 & 10 & 11 & 15 & 19 & 21 & 30 & 34 & 44 & 47 & 54 \\
\hline 4 & 3 & 10 & 12 & 15 & 18 & 16 & 18 & 20 & 25 & 26 & 30 \\
\hline 5 & $\mathrm{C}$ & 11 & 15 & 18 & 21 & 27 & 37 & 41 & 49 & 61 & 64 \\
\hline 5 & 1 & 12 & 18 & 21 & 26 & 31 & 45 & 51 & 66 & 74 & 77 \\
\hline 5 & 2 & 14 & 16 & 20 & 24 & 29 & 39 & 39 & 51 & 60 & 62 \\
\hline 5 & 3 & 11 & 11 & 12 & 14 & 16 & 16 & - & - & - & - \\
\hline 6 & $\mathrm{C}$ & 10 & 14 & 17 & 22 & 29 & 38 & 47 & 62 & 69 & 75 \\
\hline 6 & 1 & 8 & 10 & 13 & 16 & 24 & 35 & 38 & 52 & 60 & 62 \\
\hline 6 & 2 & 9 & 12 & 14 & 18 & 25 & 32 & 38 & 51 & 59 & 63 \\
\hline 6 & 3 & 11 & 14 & 16 & 20 & 27 & 32 & 37 & 48 & 52 & 51 \\
\hline 7 & $\mathrm{C}$ & 13 & 17 & 24 & 32 & 42 & 49 & 57 & 71 & 75 & 89 \\
\hline 7 & 1 & 14 & 19 & 25 & 36 & 40 & 50 & 62 & 74 & 79 & 98 \\
\hline 7 & 2 & 14 & 18 & 24 & 32 & 35 & 45 & 50 & 62 & 69 & 84 \\
\hline 7 & 3 & 13 & 16 & 21 & 28 & 29 & 34 & 36 & 39 & 44 & 52 \\
\hline 8 & C & 13 & 14 & 19 & 25 & 31 & 37 & 43 & 56 & 68 & 73 \\
\hline 8 & 1 & 13 & 13 & 18 & 23 & 30 & 35 & 42 & 52 & 66 & 72 \\
\hline 8 & 2 & 11 & 11 & 13 & 17 & 22 & 24 & 29 & 40 & 48 & 59 \\
\hline 8 & 3 & 16 & 17 & 22 & 25 & 30 & 36 & 39 & 53 & 65 & 72 \\
\hline 9 & $\mathrm{C}$ & 16 & 20 & 24 & 31 & 40 & 46 & 51 & 63 & 70 & 76 \\
\hline 9 & 1 & 16 & 21 & 26 & 30 & 39 & 47 & 52 & 63 & 69 & 74 \\
\hline 9 & 2 & 12 & 17 & 22 & 28 & 34 & 39 & 41 & 50 & 50 & 61 \\
\hline 9 & 3 & 19 & 21 & 26 & 30 & 33 & 30 & 28 & - & - & - \\
\hline 10 & C & 16 & 22 & 25 & 35 & 40 & 51 & 62 & 69 & 85 & 90 \\
\hline 10 & 1 & 12 & 17 & 20 & 25 & 29 & 36 & 50 & 62 & 71 & 71 \\
\hline 10 & 2 & 17 & 20 & 28 & 34 & 36 & 46 & 55 & 49 & 77 & 82 \\
\hline 10 & 3 & 15 & 23 & 24 & 32 & 36 & 44 & 45 & 46 & - & - \\
\hline
\end{tabular}


Figura 1.1.2: Perfis médios de resposta dos tratamentos

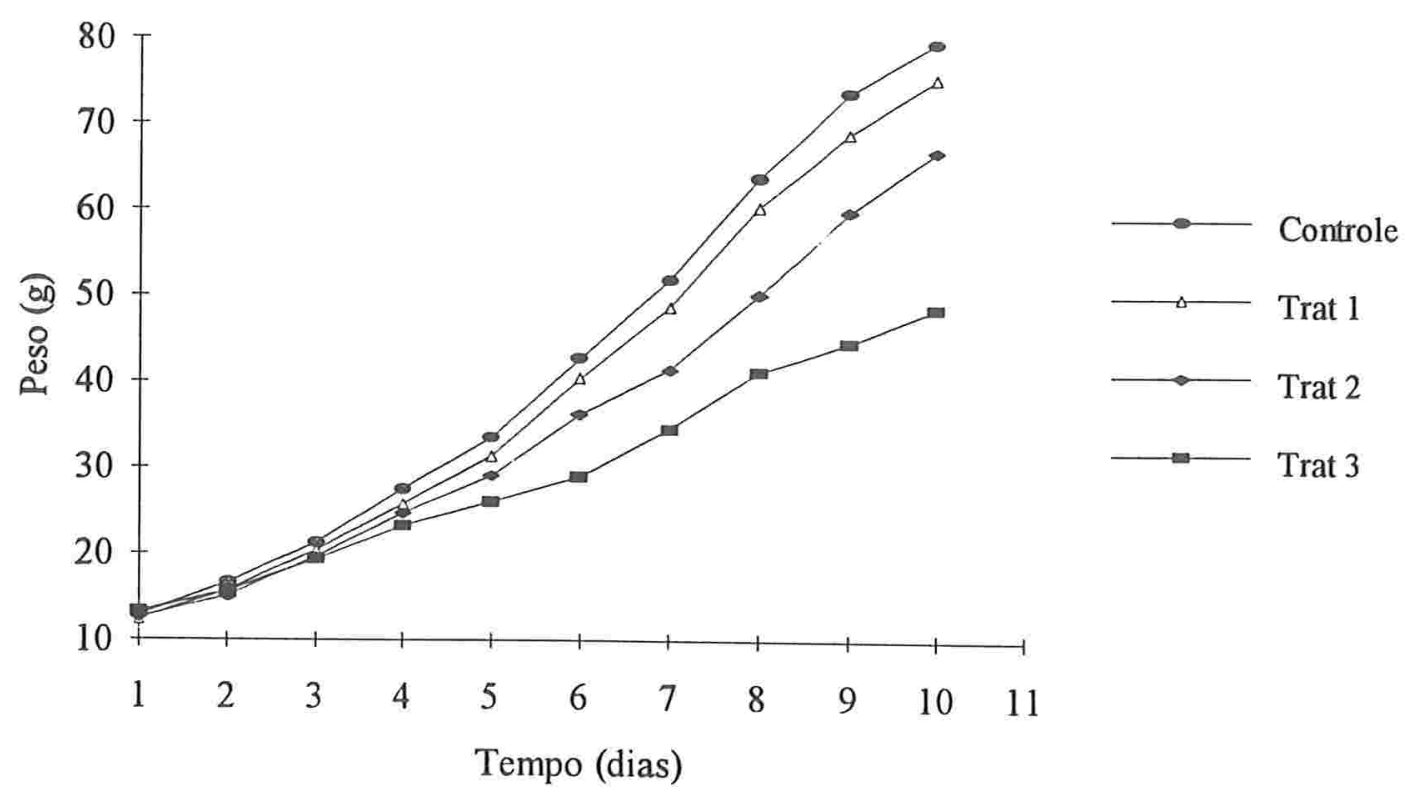

Observando-se o gráfico do peso médio dos filhotes em função do tempo (Figura 1.1.2), vemos que a velocidade do ganho de peso dos animais foi inversamente proporcional à quantidade de chumbo que ingeriram.

Exemplo 1.3 (Milliken and Johnson (1984, p.427)) Num experimento conduzido para se estudarem diferenças de variedade de sorgo quanto a um índice de área das folhas, foram utilizadas 4 variedades, plantadas segundo um planejamento com 5 blocos. Foram feitas 5 medidas desse índice para as 4 variedades em cada um dos blocos, com intervalos semanais, com início 14 dias após o surgimento dos brotos. Neste exemplo, faixa de terra é o fator Blocos com 5 níveis, as folhas são as unidades de investigação e as condições de avaliação correspondem às ocasiões em que foram tomadas as medidas do índice de área. Os dados encontram-se reproduzidos na Tabela 1.1.3 e os perfis médios de resposta para cada uma das variedades de sorgo, na Figura 1.1.3. 
Tabela 1.1.3: Indice de área das folhas para quatro variedades de sorgo

\begin{tabular}{ccccccc}
\hline Bloco & Trat & Semana 2 & Semana 3 & Semana 4 & Semana 5 & Semana 6 \\
\hline 1 & 1 & 5,00 & 4,84 & 4,02 & 3,75 & 3,13 \\
1 & 2 & 5,82 & 5,60 & 5,05 & 4,72 & 4,46 \\
1 & 3 & 5,65 & 5,97 & 5,27 & 5,07 & 4,52 \\
1 & 4 & 5,86 & 5,60 & 5,37 & 5,00 & 4,37 \\
2 & 1 & 4,42 & 4,30 & 3,67 & 3,23 & 2,83 \\
2 & 2 & 5,73 & 5,59 & 5,00 & 4,65 & 4,42 \\
2 & 3 & 5,39 & 5,49 & 5,08 & 4,87 & 4,32 \\
2 & 4 & 5,82 & 5,55 & 5,29 & 4,95 & 4,07 \\
3 & 1 & 4,42 & 4,10 & 3,46 & 3,09 & 2,82 \\
3 & 2 & 5,31 & 5,19 & 4,86 & 4,44 & 4,22 \\
3 & 3 & 5,15 & 5,28 & 4,93 & 4,67 & 4,15 \\
3 & 4 & 5,26 & 5,06 & 4,76 & 4,48 & 3,94 \\
4 & 1 & 4,01 & 3,89 & 3,21 & 2,89 & 2,56 \\
4 & 2 & 4,92 & 4,66 & 4,56 & 4,16 & 3,99 \\
4 & 3 & 4,50 & 4,89 & 4,74 & 4,49 & 4,10 \\
4 & 4 & 4,87 & 4,75 & 4,55 & 4,33 & 3,83 \\
5 & 1 & 3,36 & 3,10 & 2,67 & 2,47 & 2,16 \\
5 & 2 & 3,96 & 3,86 & 3,50 & 3,13 & 2,95 \\
5 & 3 & 3,75 & 3,74 & 3,55 & 3,28 & 3,00 \\
5 & 4 & 3,96 & 3,76 & 3,56 & 3,18 & 2,96 \\
& & & & & & \\
\hline & & & & & & \\
\hline
\end{tabular}


Figura 1.1.3:Perfis médios de resposta das diferentes variedades de sorgo

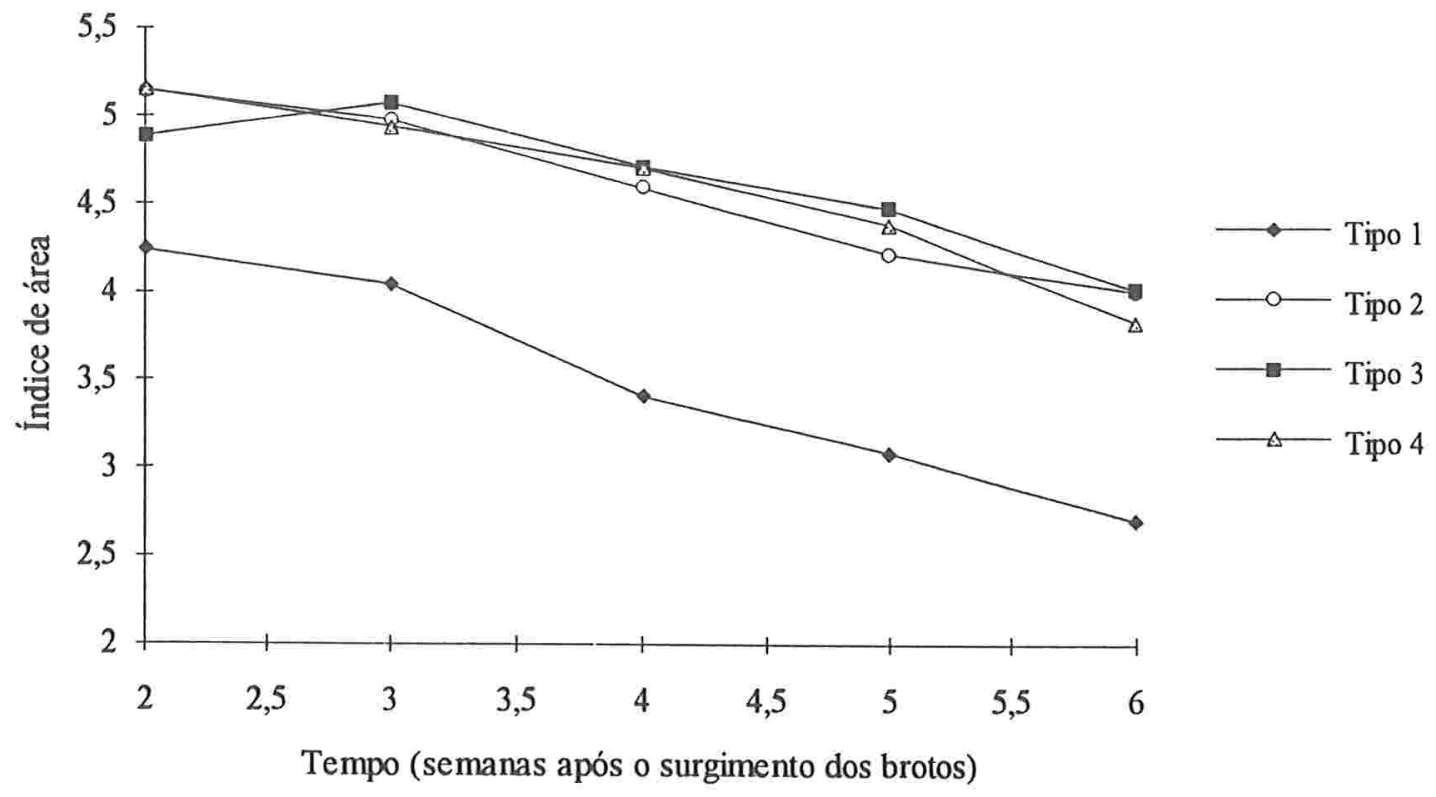

Em geral, não se tem interesse no efeito de blocos. No entanto, se eles forem ignorados na análise, pode haver um comprometimento dos resultados, dado que a variabilidade devida a blocos estará sendo incorporada ao erro. A extensão das técnicas de Análise de Perfis clássica para dados provenientes de planejamentos em blocos, entretanto, não é direta. A finalidade deste trabalho é discutir os principais aspectos da Análise de Perfis no caso de blocos completos aleatorizados.

No Capítulo 2 introduzimos a notação a ser utilizada e apresentamos uma breve resenha das parametrizações e dos principais modelos para a estrutura de covariância utilizados em Análise de Perfis. No Capítulo 3 abordamos técnicas para a Análise de Perfis para experimentos com planejamento em blocos, sejam estes fixos ou aleatórios. No Capítulo 4 apresentamos os resultados e as conclusões das análises dos dados do Exemplo 1.1 ilustrando, dessa forma, os procedimentos propostos, bem como sugestões para futuras pesquisas. Os programas utilizados nas análises e os demais detalhes técnicos encontram-se no Apêndice B. 


\section{Capítullo 2}

\section{Análise de Perfis Para Experimentos Completamente Alleatorizados}

\subsection{Introdução}

Antes de abordarmos o assunto principal deste trabalho, ou seja, a inclusão do efeito de blocos na Análise de Perfis, vamos rever métodos clássicos, bem como algumas propostas mais recentes para o caso de dados provenientes de um planejamento completamente aleatorizado.

Suponhamos uma situação genérica onde $p$ respostas foram coletadas de $n$ unidades de investigação, agrupadas segundo $t$ tratamentos. Vamos admitir que os dados estejam arranjados como na Tabela 2.1.1. Desse modo, a resposta do $j$-ésimo indivíduo submetido ao $i$-ésimo tratamento na $k$-ésima condição de avaliação será denotada por $y_{i j k}$ e $y_{i j}$ representará o vetor de respostas (perfil de respostas) desse indivíduo:

$$
y_{i j}=\left(y_{i j 1} y_{i j 2}, \ldots, y_{i j p}\right)^{t}
$$


Tabela 2.1.1: Estrutura básica dos dados

Unidade de observação

\begin{tabular}{cccccc}
\hline Tratamento & $\begin{array}{c}\text { Unidade de } \\
\text { investigação }\end{array}$ & $t_{1}$ & $t_{2}$ & $\cdots$ & $t_{p}$ \\
\hline 1 & 1 & $y_{111}$ & $y_{112}$ & $\ldots$ & $y_{11 p}$ \\
1 & 2 & $y_{121}$ & $y_{122}$ & $\ldots$ & $y_{12 p}$ \\
$\vdots$ & $\vdots$ & $\vdots$ & $\vdots$ & $\cdots$ & $\vdots$ \\
1 & $n_{1}$ & $y_{1 n_{1} 1}$ & $y_{1 n_{1} 2}$ & $\ldots$ & $y_{1 n_{1} p}$ \\
2 & 1 & $y_{211}$ & $y_{212}$ & $\ldots$ & $y_{21 p}$ \\
2 & 2 & $y_{221}$ & $y_{222}$ & $\ldots$ & $y_{22 p}$ \\
$\vdots$ & $\vdots$ & $\vdots$ & $\vdots$ & $\cdots$ & $\vdots$ \\
2 & $n_{2}$ & $y_{2 n_{2} 1}$ & $y_{2 n_{2} 2}$ & $\ldots$ & $y_{2 n_{2 p} p}$ \\
$\vdots$ & $\vdots$ & $\vdots$ & $\vdots$ & $\vdots$ & $\vdots$ \\
$t$ & 1 & $y_{t 11}$ & $y_{t 12}$ & $\ldots$ & $y_{t 1 p}$ \\
$t$ & 2 & $y_{t 21}$ & $y_{122}$ & $\ldots$ & $y_{t 2 p}$ \\
$\vdots$ & $\vdots$ & $\vdots$ & $\vdots$ & $\cdots$ & $\vdots$ \\
$t$ & $n_{t}$ & $y_{t n_{t} 1}$ & $y_{t n_{t} 2}$ & $\cdots$ & $y_{t n_{t} p}$ \\
\hline
\end{tabular}

Adotaremos para $y_{i j}$ o modelo

$$
\mathbf{y}_{i j}=\mathbf{X}_{i j} \beta_{i}+\varepsilon_{i j}
$$

onde $\mathbf{X}_{i j}$ é uma matriz de especificação $p \times q(q<n p), \beta_{i}$ é um vetor de dimensão ( $q$ $\times 1)$ de parâmetros de regressão desconhecidos e os $\varepsilon_{i j}$ são erros independentes com distribuição $\mathrm{N}\left(0, \Sigma_{i j}\right)$. Assume-se ainda que os elementos de $\Sigma_{i j}$ para $i=1, \ldots, t \mathrm{e}$ $j=1, \ldots, n_{i}, n=\sum_{i=1}^{t} n_{i}$, são funções conhecidas de $r$ parâmetros de covariância desconhecidos contidos em um vetor $\theta$.

O modelo (2.1.2) para todo o conjunto de dados pode ser escrito na forma

$$
\boldsymbol{Y}=\boldsymbol{X} \beta+\varepsilon
$$


onde

$$
Y=\left[\begin{array}{c}
y_{11} \\
y_{12} \\
\vdots \\
y_{t n_{t}}
\end{array}\right], \quad \varepsilon=\left[\begin{array}{c}
\varepsilon_{11} \\
\varepsilon_{12} \\
\vdots \\
\varepsilon_{t n_{t}}
\end{array}\right], \quad \beta=\left[\begin{array}{c}
\beta_{1} \\
\beta_{2} \\
\vdots \\
\beta_{q}
\end{array}\right]
$$

e $X=\left(X_{11}^{t}, X_{12}^{t}, \ldots, X_{t n_{t}}^{t}\right)^{t}$ é uma matriz de especificação de posto $q<n p$.

Dessa forma, $\mathrm{E}(Y)=X \beta$ e $\operatorname{Var}(Y)=\operatorname{diag}\left(\Sigma_{11}(\theta), \Sigma_{21}(\theta), \ldots, \Sigma_{n, t}(\theta)\right)$, onde $\operatorname{diag}\left(A_{1}, \ldots, A_{\mathrm{n}}\right)$ representa uma matriz diagonal em blocos com as matrizes $A_{1}, \ldots, A_{\mathrm{n}}$ ao longo da diagonal principal.

As colunas da matriz de especificação $X$ podem estar associadas aos fatores que definem a estrutura das subpopulações, isto é, Tratamento, ao fator Tempo e a possíveis covariáveis.

\subsection{Medidas Resumo}

Entendemos por medida resumo qualquer transformação que produza uma redução na dimensão dos vetores de respostas dos tratamentos. A utilização desse recurso pode acarretar perda de parte da informação contida nos dados mas, por outro lado, permitenos responder a questões de interesse específico utilizando técnicas com as quais já estamos familiarizados, como a Análise de Variância com um fator, por exemplo. Um outro atrativo para o uso de medidas resumo é o fato de acomodarem dados incompletos. Rowell and Walters (1976) propõem como medidas resumo funções lineares da variável resposta com relação ao tempo, sendo a escolha dessas funções sugerida principalmente pelos interesses da pesquisa. Em curvas de crescimento, por exemplo, o uso de polinômios ortogonais pode ser apropriado (Kowalski and Guire (1974)). Wishart (1938), ao analisar os dados de um experimento na área de nutrição suína utilizou como medidas resumo os coeficientes de regressão correspondentes a cada animal.

Algumas funções do perfil de respostas, não necessariamente ortogonais, podem fornecer informações suficientes para a caracterização dos tratamentos (em geral, consideram-se transformações que resultem em vetores unidimensionais e, eventualmente, em vetores bi ou tridimensionais). Vamos tomar o Exemplo 1.2 para efeito de ilustração; naquele caso, poderia haver interesse na construção de uma medida 
da tendência linear do peso dos animais ao longo das ocasiões de pesagem. Assim, o contraste

$$
W_{i j}=-3 y_{i j 1}-y_{i j 2}+y_{i j 3}+3 y_{i j 4}
$$

seria calculado para cada uma das unidades observacionais e os valores obtidos comparados através de uma análise de variância com um único fator, testando-se assim a . hipótese de homogeneidade desses contrastes para todas as dietas. Ainda com relação ao Exemplo 1.2, o interesse principal poderia residir na resposta obtida ao término do período de estudo, ou seja

$$
W_{i j}=y_{i j p}
$$

$i=1, \ldots, t ; j=1, \ldots, n_{t}$. A análise desse tipo de medida (conhecida como ponto final) é recomendada para se compararem diferenças entre tratamentos para os quais se espera uma resposta monotônica crescente (ou decrescente) durante o período de observação. Outras estratégias envolvem o uso do nível máximo de resposta, $\max y_{i j k}(i=1, \ldots, t ; j=1$, $\left.\ldots, n_{t} ; k=1, \ldots, p\right)$ ou a média entre condições de avaliação,

$$
W_{i j}=\frac{1}{p} \sum_{k=1}^{p} y_{i j k}
$$

Esta última, ao contrário do ponto final e da resposta máxima, faz uso de toda a informação dos perfis. Em situações onde os intervalos entre as observações apresentam diferentes comprimentos, é preciso levar em conta a informação contida nesses espaçamentos. Neste caso, uma medida adequada é a Área sob a Curva (ASC), que é aplicável tanto na situação de dados balanceados em relação ao tempo como na de dados desbalanceados (neste último caso, é necessário escolher um período de tempo comum a todos os tratamentos). Ver Grande et al. (1992) para um exemplo onde se usa a ASC como medida resumo.

Na ausência de informações $a$ priori acerca da relação entre a variável e o tempo, os contrastes podem ser escolhidos com base empírica. Os polinômios ortogonais em geral dão conta de porções substanciais da variação do erro, porém seus coeficientes são freqüentemente difíceis de interpretar. 


\subsection{Modelo Multivariado}

Para se representar o modelo multivariado é mais conveniente usar uma notação ligeiramente diferente daquela apresentada na Seção 2.1. Nesta notação, o conjunto de todas as observações é apresentado na forma matricial:

$$
Y=X \beta+E
$$

onde $Y$ é a matriz $(n \times p)$ em que cada linha corresponde a uma unidade de investigação e cada coluna corresponde a uma das medidas repetidas, $X$ é uma matriz de especificação de posto $q$, com $n$ linhas e $r$ colunas, $\beta$ é uma matriz onde cada coluna $(r \times 1)$ de parâmetros desconhecidos corresponde a uma particular medida repetida e $E$ é uma matriz de dimensões $(n \times p)$ de erros aleatórios não-observáveis. Assume-se que as linhas de $\varepsilon$ são independentes e identicamente distribuídas com distribuição $\mathrm{N}(0, \Sigma)$, onde $\Sigma$ é uma matriz simétrica positiva-definida sem estrutura específica. Os elementos nas colunas de $\varepsilon$ podem ser correlacionados.

Relativamente a esse esquema, podemos testar hipóteses de interesse, como aquelas descritas na Seção 1.2, escrevendo-as na forma

$$
H_{0}: C \beta U=0 \text { versus } H_{a}: C \beta U \neq 0
$$

onde $C$ é uma matriz $(c \times r)$ de posto $c$ e $U$ é uma matriz $(p \times u)$ de posto $u$.

Utilizando o princípio da União-Intersecção de Roy (1953) ou o princípio da razão de verossimilhança de Wilks (1932), podem ser obtidas estatísticas para se testar a hipótese (2.3.2). Tais estatísticas são funções das raízes características $\lambda_{i}(i=1, \ldots, s)$ da matriz $H G^{-1}$, onde

$$
H=U^{t} \hat{\beta} C^{t}\left[C\left(X^{t} X\right)^{-1} C^{t}\right]^{-1} C \hat{\beta} U
$$

e

$$
\mathbf{G}=\mathbf{U}^{t} \mathbf{Y}^{t}\left[\mathbf{I}-\mathbf{X}\left(\mathbf{X}^{t} \mathbf{X}\right)^{-1} \mathbf{X}^{t}\right] \mathbf{Y} \mathbf{U}
$$

com

$$
\hat{\beta}=\left(X^{t} X\right)^{-1} X^{t} Y \text {. }
$$

Fazendo $\theta_{i}=\lambda_{i}\left(1+\lambda_{i}\right)^{-1}$, as estatísticas de teste mais comuns são: 
- $\quad \lambda$ de Wilks $=\Pi_{i=1}^{s}\left(1-\theta_{i}\right)$;

- Traço de Pillai $=\Sigma_{i=1}^{s} \theta_{i}$;

- Traço de Lawley-Hotelling $=\sum_{i=1}^{s} \theta_{i}\left(1-\theta_{i}\right)^{-1} \quad$ e

- Raiz máxima de Roy $=\max \left(\theta_{i}\right)$

As distribuições exatas dessas estatísticas sob a hipótese nula dependem unicamente dos . parâmetros $m_{1}=(|u-c|-1) / 2, m_{2}=(n-q-u-1) / 2$ e $s=\min (u, c)$; tabelas dessas distribuições podem ser encontradas em Timm (1975) ou Morrison (1976), por exemplo. Aproximações assintóticas através de distribuições qui-quadrado ou $\mathrm{F}$ são consideradas em Seber (1984), entre outros.

Como nenhuma suposição é feita sobre a estrutura de covariância, todos os $p(p+1) / 2$ parâmetros da matriz de covariâncias precisam ser estimados. Além disso, em situações com poucas unidades de investigação em relação ao número de medidas repetidas, a matriz de covariâncias amostral será singular e as estatísticas de teste não estarão nem mesmo definidas. Assim, a desvantagem do modelo de Análise Multivariada de Perfis é a sua possível ineficiência nesse caso, o que torna o seu uso desaconselhável. Os módulos ANOVA e GLM do programa SAS, 4V do programa BMDP, MANOVA do SPSS e as subrotinas MLM.AJU e MLM.HIP (Andrade et al., 1988) implementadas no módulo CM do SOC fornecem os testes.

Esses pacotes permitem testar outros tipos de hipóteses, além das hipóteses de inexistência de efeito de Tempo, Tratamento e interação entre Tempo e Tratamento. 


\subsection{Modelo Misto Univariado (tipo "SPLIT-PLOT")}

Em Análise de Perfis é comum adotar-se um modelo misto univariado para representar cada uma das observações $y_{i j k}$ Neste modelo, Tratamento e Tempo são considerados como dois fatores fixos completamente cruzados $\mathrm{e}$ as unidades de investigação como um fator aleatório hierárquico relativamente a Tratamento. Assim, , utilizando uma parametrização identificável, baseada em desvios médios com restrições, podemos escrever:

$$
\begin{aligned}
& y_{i j k}=\mu+\tau_{i}+\pi_{j(i)}+\alpha_{k}+(\tau \alpha)_{i k}+\varepsilon_{i j k} \\
& i=1, \ldots, t ; j=1, \ldots, n_{i} ; k=1, \ldots, p .
\end{aligned}
$$

$\operatorname{com} \sum_{i=1}^{t} \tau_{i}=\sum_{k=1}^{p} \beta_{k}=\sum_{i=1}^{t}(\tau \beta)_{i k}=\sum_{k=1}^{p}(\tau \beta)_{i k}=0$ onde $\mu$ representa a média geral, $\tau_{i}$ o "efeito" do $i$-ésimo tratamento, $\pi_{j(i)}$ é o "efeito" do j-ésimo indivíduo dentro do $i$-ésimo tratamento, $\gamma_{k} 0$ "efeito" da $k$-ésima condição de avaliação, e $(\tau \alpha)_{i k}$ a interação entre $0 i$ ésimo tratamento e a $k$-ésima condição de avaliação. Os $\pi_{j(i)}$ representạm os efeitos aleatórios individuais e os $\varepsilon_{i j k}$ representam os erros de medida associados às condições de avaliação. Além disso, supomos que $\pi_{j(i)}$ e $\varepsilon_{i j k}$ são variáveis aleatórias independentemente distribuídas com média zero e variâncias $\sigma_{\pi}^{2}$ e $\sigma_{\varepsilon}^{2}$, respectivamente.

A análise dos dados sob o modelo misto univariado corresponde à análise tipo "SPLIT-PLOT" usual, onde os indivíduos representam as parcelas ("plots") e as ocasiões de observação, as subparcelas ("subplots").

Dado o modelo (2.4.1), temos $\mathbf{E}\left(y_{i j k}\right)=\mu+\tau_{i}+\alpha_{k}+(\tau \alpha)_{i k} \mathrm{e}$

$$
\operatorname{Cov}\left(y_{i j}\right)=\Sigma=\sigma^{2}\left[\begin{array}{ccccc}
1 & \rho & \rho & \cdots & \rho \\
\rho & 1 & \rho & \cdots & \rho \\
\rho & \rho & 1 & \cdots & \rho \\
\vdots & \vdots & \vdots & \ddots & \rho \\
\rho & \rho & \rho & \cdots & 1
\end{array}\right]
$$

onde $\sigma^{2}=\sigma_{\pi}^{2}+\sigma_{\varepsilon}^{2}$ e $\rho=\sigma_{\pi}^{2} /\left(\sigma_{\pi}^{2}+\sigma_{\varepsilon}^{2}\right)$, o que implica $0<\rho<1$. Nesse caso, dizemos que $\Sigma$ segue o padrão de uniformidade. 
Essa condição implica que as variâncias dos elementos $y_{i j}$ são todas iguais e que as correlações entre dois elementos quaisquer de $y_{i j}$ são também constantes. Tal suposição é mais adequada para situações em que as unidades observacionais são alocadas ao acaso às $p$ condições de avaliação. A característica que distingue os planejamentos longitudinais dos planejamentos do tipo "SPLIT-PLOT" é a forma sistemática (ao longo do tempo, por exemplo) com que as condições de avaliação são alocadas às unidades de investigação; , em planejamentos do tipo "SPLIT-PLOT" as condições de avaliação ("subplots") são atribuídas aleatoriamente às unidades de investigação. Em estudos longitudinais as observações são feitas seqüencialmente e espera-se que a correlação entre duas observações tomadas em ocasiões próximas seja maior do que a correlação entre observações tomadas em ocasiões mais distantes, o que torna (2.4.2) pouco realista.

Huynh and Feldt (1970) e Rouanet and Lépine (1970), em trabalhos independentes, mostraram que na realidade os testes $\mathrm{F}$ considerados para o modelo misto univariado são válidos sob condições mais gerais para a matriz $\Sigma$. Basta que $\Sigma$ satisfaça à condição de esfericidade, ou seja

$$
\sigma_{k k^{\prime}}=\left\{\begin{array}{lll}
\gamma_{k}+\gamma_{k^{\prime}}+\lambda & \text { se } & k=k^{\prime} \\
\gamma_{k}+\gamma_{k^{\prime}} & \text { se } & k \neq k^{\prime}
\end{array}\right.
$$

ou, em termos matriciais

$$
\Sigma=\lambda \mathbb{I}_{p}+\gamma \mathbf{1}_{p}{ }^{t}+\mathbf{1}_{p} \gamma^{t}
$$

onde $\mathbb{I}_{p}$ denota uma matriz identidade de ordem $p, \mathbf{1}_{p}$ um vetor $(p \times 1)$ com todos os elementos iguais a $1, \lambda$ é uma constante positiva e $\gamma=\left(\gamma_{1}, \gamma_{2}, \ldots, \gamma_{p}\right)^{t}$ é um vetor de parâmetros desconhecidos. Essa condição equivale a especificar que as variâncias das diferenças entre pares de erros, tais como $\xi_{i j k}-\xi_{i j k^{\prime}}$ sejam iguais para todos $k$ e $k^{\prime}, k \neq$ $k^{\prime}$, permitindo variâncias desiguais e correlações desiguais. Se as variâncias forem todas iguais, tal condição é equivalente à imposição do padrão de uniformidade. $\mathrm{O}$ padrão de uniformidade e aquele associado a erros independentes são, portanto, casos particulares do padrão de circularidade. Isso pode ser facilmente comprovado observando-se, por exemplo, as seguintes matrizes: 


$$
\begin{aligned}
& \Sigma_{1}=\left[\begin{array}{lll}
1,4 & 1,1 & 0,8 \\
1,1 & 1,6 & 0,9 \\
0,8 & 0,9 & 1,0
\end{array}\right] \\
& \Sigma_{2}=\left[\begin{array}{lll}
1,4 & 1,0 & 1,0 \\
1,0 & 1,4 & 1,0 \\
1,0 & 1,0 & 1,4
\end{array}\right] \\
& \Sigma_{3}=\left[\begin{array}{lll}
0,4 & 0,0 & 0,0 \\
0,0 & 0,4 & 0,0 \\
0,0 & 0,0 & 0,4
\end{array}\right]
\end{aligned}
$$

A matriz $\Sigma_{1}$ satisfaz à condição de circularidade (2.11), com $\gamma_{1}=0,5, \gamma_{2}=0,6, \gamma_{3}=0,3 \mathrm{e}$ $\lambda=0,4$. Fazendo $\gamma_{1}=\gamma_{2}=\gamma_{3}=0,5$ e mantendo $\lambda=0,4$, obtemos $\Sigma_{2}$, que além de satisfazer a (2.4.3), segue também o padrão (2.4.2). $\Sigma_{3}$ é obtida no caso particular em que $\gamma_{1}=\gamma_{2}=\gamma_{3}=0$ e corresponde ao caso de independência das observações.

Pode-se mostrar que (2.4.4) é equivalente a

$$
\mathbb{P} \sum \mathbf{P}^{t}=\lambda \mathbb{I}
$$

onde $\mathbf{P}$ é uma matriz contraste ortonormal de dimensões $\mathrm{p} \times(\mathrm{p}-1)$ e $\lambda$ é um dos $p+1$ parâmetros que definem o padrão de circularidade. Mauchly (1940) obteve um teste para a validade deste padrão utilizando a estatística

$$
\mathrm{M}=-\left(-n-1-\frac{2 p^{2}-3 p+3}{6(p-1)}\right) \log \mathrm{W}
$$

com

$$
\mathrm{W}=\frac{(p-1)^{p-1}\left|\mathbf{P S P} \mathbf{P}^{t}\right|}{\left(\operatorname{tr} \mathbf{P S} \mathbf{P}^{t}\right)^{p-1}} .
$$

Quando $\Sigma$ segue o padrão de circularidade, $\mathrm{M}$ segue assintoticamente uma distribuição qui-quadrado com $p(p-1) / 2-1$ graus de liberdade.

Quando $\Sigma$ não satisfaz à condição de circularidade, a análise "SPLIT-PLOT" usual deixa de ser válida. Entretanto, ainda continuam sendo válidos os testes relativos às diferenças entre tratamentos, tendo em vista que estão associadas somente a comparações entre indivíduos. 
Vários autores propuseram medidas para acomodar desvios relativos a esse padrão de circularidade. Box (1954) sugeriu um teste F conservativo com graus de liberdade corrigidos por um fator dado por

$$
\varepsilon=\left[\operatorname{tr}\left(\mathbb{P}^{t} \sum \mathbb{P}\right)\right]^{2} /\left[(p-1) \operatorname{tr}\left(\mathbb{P}^{t} \sum \mathbb{P}^{2}\right)\right]
$$

para testar efeitos relacionados com o fator Tempo. Como $\varepsilon$ não é conhecido na prática, é ' preciso estimá-lo a partir da matriz de covariância amostral. Box (1954) e Geisser and Greenhouse (1958) mostraram que $(p-1)^{-1}<\varepsilon<1$ para qualquer matriz de covariância (o uso dos extremos 1 ou $(p-1)^{-1}$ como valores para $\varepsilon$ pode fazer com que o teste seja muito liberal ou muito conservativo, respectivamente). Greenhouse and Geisser (1959) desenvolveram um procedimento em três estágios onde nem sempre é necessário o cálculo da estimativa do fator de correção; Huynh and Feldt (1976) desenvolveram estimativa que, em certas situações é melhor do que a de Greenhouse and Geisser (1959). Os pacotes estatísticos BMDP(2V,4V), SAS(ANOVA, GLM) e SPSS(MANOVA) permitem fazer testes de esfericidade para explorar a legitimidade do Modelo Misto Univariado e incorporam as correções de Huynh and Feldt (1976) e de Greenhouse and Geisser (1959), além de permitirem que se testem outras hipóteses além das hipóteses padrão. 


\subsection{Outros modelos}

$\Sigma$. Nesta seção apresentamos outras possíveis estruturas para a matriz de covariâncias

\subsubsection{Modelo com Estrutura de Antedependência}

Diz-se que um conjunto de $p$ variáveis aleatórias ordenadas tem estrutura de antedependência de ordem $r$ se a $k$-ésima variável $(k>r)$, dadas as $r$ anteriores, é independente de qualquer outra variável predecessora (Gabriel (1961),(1962)). Sob a estrutura de antedependência de ordem $r$, a inversa da matriz de covariância das $p$ variáveis tem elementos não-nulos somente na diagonal principal e nas $r$ diagonais imediatamente superiores e imediatamente inferiores à diagonal principal (uma demonstração para esta afirmativa pode ser encontrada em Gabriel (1962)). Sob a hipótese de normalidade, tal estrutura de covariância equivale à suposição da estrutura de antedependência correspondente. Independência $(r=0)$ e dependência geral $(r=p$-1) são casos especiais da estrutura de antedependência. Gabriel (1962) obteve o teste da razão de verossimilhança sob normalidade para comparar os ajustes das estruturas de ordens $r \mathrm{e}$ $s, r<s$. Kenward (1987) obteve o mesmo teste utilizando Análise de Covariância. Neste mesmo artigo, ele aplica esses conceitos à Análise de Perfis.

Suponhamos que um conjunto de dados organizados como na Tabela 2.1, sendo $y_{k}$ o vetor que contém as $n$ observações referentes ao $k$-ésimo tempo. Sob a suposição de uma estrutura de antedependência de ordem $r$, a verossimilhança para esses dados pode ser subdividida em $p$ componentes independentes, onde o $k$-ésimo componente consiste na verossimilhança de $y_{k}$ condicionada a $y_{k-r_{k}}, \ldots, y_{k-1}$, onde $\mathrm{r}_{k}=\min (k-1, r)$. Essa verossimilhança maximizada pode ser expressa como (Kenward (1987))

$$
L_{0}=\prod_{k=1}^{p} C_{k} d_{k}^{-n / 2}
$$

onde $C_{k}$ é uma constante e $d_{k}$ é a soma de quadrados residual da Análise de Covariância tendo $y_{k}$ como variável resposta e $y_{k-r_{k}, \ldots, y_{k-1}}$ como covariáveis. Analogamente, a verossimilhança maximizada sob a suposição de uma estrutura de antedependência de ordem $s(s>r)$ pode ser expressa como 


$$
L_{1}=\prod_{k=1}^{p} C_{k} e_{k}^{-n / 2}
$$

onde $e_{k}$ é a soma de quadrados residual da Análise de Covariância tendo como variável resposta $y_{k} \mathrm{e} y_{k-s_{k}}, \ldots, y_{k-1}$ como covariáveis. Sob a hipótese de que a estrutura de antedependencia é de ordem $\mathrm{r}$, a razão $-2 \ln \left(L_{0} / L_{1}\right)$ tem uma distribuição assintótica $\chi^{2}$ com $\sum_{i=r+2}^{p} t_{i}$ graus de liberdade, onde $t_{i}=s_{i}-r_{i}$ (Kenward (1987)). A partir desses * resultados, podem-se comparar estruturas com diferentes ordens obtendo-se, assim, a estrutura com a menor ordem possível que forneça um ajuste adequado para os dados. Determinada tal estrutura, podem-se comparar os perfis médios de resposta. Consideremos um contraste entre tratamentos cujo valor na $k$-ésima condição de avaliação denotaremos por $W_{k}$, e para o qual desejamos testar a hipótese nula $\mathrm{H}_{0}: W_{1}=$ $W_{2}=\ldots=W_{p}=0$. Gabriel (1961) obteve o teste da razão de verossimilhança, sob normalidade, da hipótese $\mathrm{H}_{0}$ contra a alternativa $\mathrm{H}_{1}: W_{i} \neq W_{j}$ para pelo menos um par (i, j). É possível também estabelecer o primeiro instante em que ocorreu diferença entre os tratamentos através de uma seqüência de testes $\mathrm{F}$ (ou testes $\mathrm{t}$, para comparações com um grau de liberdade) da Análise de Covariância tendo $y_{i}$ como variável resposta e $y_{i-r_{i}, \ldots, y_{i-1}}$ como covariáveis. Kenward (1987) estendeu este método para o caso de múltiplos vetores de resposta. Contudo, este modelo não é recomendável para situações onde se têm objetivos específicos, tais como aquelas onde há interesse na comparação de certas características dos perfis (nível médio, máximo nível alcançado, etc) entre tratamentos. É dirigido particularmente para estudos com caráter exploratório, onde não se tem idéia da existência ou não de diferenças entre os tratamentos ou da forma que tomam, quando existirem.

\subsubsection{Modelo com Estrutura Autoregressiva}

Alguns autores (Albohali (1983), LaVange and Helms (1983), por exemplo) sugerem a incorporação de métodos utilizados em Séries Temporais para modelar a estrutura de covariância. Em particular, o modelo autoregressivo de primeira ordem discutido a seguir tem sido considerado com essa finalidade e, nesse sentido, os elementos de $\varepsilon_{i j}$ em (2.1.2) são dados por 


$$
\varepsilon_{i j k}=\rho \varepsilon_{i j(k-1)}+u_{i j k}
$$

$k=1, \ldots, p, \operatorname{com} u_{i j k^{\prime}} \sim \mathrm{N}\left(0, \sigma_{u}^{2}\right), \operatorname{Cov}\left(u_{i j k}, u_{i j k^{\prime}}\right)=0 \quad\left(k \neq k^{\prime}\right)$ e a condição inicial $\varepsilon$ ijo i.i.d. $\mathrm{N}\left(0, \sigma_{u}^{2}\left(1-\rho^{2}\right)^{-1}\right)$, para todo $(i, j)$. Através de substituições sucessivas pode-se mostrar que a matriz de covariância "intra" indivíduo correspondente a esse modelo é dada por

$$
\Sigma=\frac{\sigma_{u}^{2}}{\left(I-\rho^{2}\right)}\left[\begin{array}{ccccc}
1 & \rho & \rho^{2} & \cdots & \rho^{p-1} \\
\rho & 1 & \rho & \cdots & \rho^{p-2} \\
\rho^{2} & \rho & 1 & \cdots & \rho^{p-3} \\
\vdots & \vdots & \vdots & \ddots & \vdots \\
\rho^{p-1} & \rho^{p-2} & \rho^{p-3} & \cdots & 1
\end{array}\right] .
$$

Como já foi dito anteriormente, em estudos longitudinais espera-se que a correlação entre observações de uma mesma unidade observacional decresça à medida que a distância entre elas aumenta. A estrutura de correlação serial acima incorpora esse padrão de correlações decrescentes. Albohali (1983) propõe um método que usa uma estimativa de máxima verossimilhança para $\rho$ e os estimadores de mínimos quadrados dos $\varepsilon_{i j k}$ para "filtrar" os dados; em outras palavras, esse método tenta remover a autocorrelação entre os intervalos de tempo e executar a análise da variação "entre" indivíduos. Feito, isso, uma Análise de Perfis utilizando qualquer outro método usual é aplicada aos dados filtrados para completar a análise dos fatores "intra" indivíduos. Ainda com relação aos modelos que incorporam séries temporais à estrutura de covariância, LaVange and Helms (1983) usaram o método dos momentos para a estimação dos parâmetros do modelo (2.1.2) onde $\varepsilon_{i j}$ segue um processo $\operatorname{AR}(1)$ ou MA(q).

Uma crítica a ser feita ao modelo com estrutura autoregressiva está na suposição de que a variância se mantém constante no Tempo, o que nem sempre reflete a realidade. 


\subsubsection{Modelo de Efeitos Aleatórios}

Os modelos de efeitos aleatórios constituem uma alternativa interessante para a análise de dados longitudinais. Assume-se para o indivíduo $i$ submetido ao tratamento $j$, por exemplo, que as observações são tomadas nos instantes $t_{i, 1}, \ldots, t_{i, p}$; a situação de dados desbalanceados é, portanto, acomodada (caso o leitor deseje informações mais específicas a esse respeito, pode consultar Laird and Ware (1982) ou Andreoni (1989), por exemplo). Freqüentemente, os parâmetros relativos a indivíduos têm uma interpretação natural que pode ser de relevância para os objetivos do estudo e as suas estimativas podem ser usadas para análises exploratórias.

Utilizando idéias introduzidas por Harville (1977), Laird and Ware (1982) propuseram uma família de modelos para dados com medidas repetidas. Trata-se de um modelo em dois estágios: parâmetros populacionais, efeitos individuais e variação "intra" indivíduos são introduzidos no primeiro estágio e a variação "entre" indivíduos, no segundo estágio. Especificamente, denotemos por $y_{i j}$ o vetor $(p \times 1)$ contendo as $p$ medidas da (ij)-ésima unidade experimental. O modelo considerado é

$$
y_{i j}=X_{i j} \beta+Z_{i j} b_{i j}+\omega_{i j}
$$

onde $X_{i j}$ e $Z_{i j}$ são matrizes de especificação conhecidas de dimensões $(p \times q)$ e $(p \times k)$, respectivamente, $\beta$ é um vetor de parâmetros fixos a ser estimado e $b_{i j}$ e $\omega_{i j}$ são vetores aleatórios independentes com distribuição $\mathrm{N}\left(0, \Sigma_{b}\right)$ e $\mathrm{N}\left(0, \Sigma_{w}\right)$, respectivamente.

Laird and Ware (1982) concentraram-se na situação onde os erros "intra" indivíduos são independentes, ou seja, $\Sigma_{w}=\sigma^{2} \mathbf{I}_{p}$, implicando

$$
\operatorname{Var}\left(y_{i j}\right)=\Sigma_{i j}=\mathbf{Z}_{i j} \Sigma_{b} \mathbf{Z}_{i j}^{t}+\sigma^{2} \mathbf{I}_{p}
$$

Note que, condicionalmente aos efeitos aleatórios $b_{i j}$, supõe-se que as observações de um dado indivíduo são independentes. Isto sugere que nesse caso uma escolha criteriosa dos termos $\beta$ pode ser necessária para representar adequadamente a estrutura de covariância não-condicional de $y_{i, j}$. Dessa forma, pode ser interessante incorporar, além da estrutura de efeitos aleatórios em (2.5.5), a possibilidade de que os erros individuais $\omega_{i j}$ sejam, por exemplo, autocorrelacionados. 
Para o modelo (2.5.5), com, $\Sigma_{\omega}=\sigma^{2} I_{p}$, Laird and Ware (1982) discutem detalhadamente como usar o algoritmo EM para obter as estimativas de máxima verossimilhança (EMV) e as estimativas de máxima verossimilhança restrita (EMVR) dos componentes de $\Sigma_{b}$ e $\sigma^{2}$. Mais recentemente, Laird, Lange and Stram (1987) dão continuidade ao estudo da aplicação do algoritmo EM para esse modelo de efeitos aleatórios. Ainda nesse trabalho, consideram o modelo (2.5.5) no contexto de ajuste de . curvas de crescimento com dados balanceados e completos e apresentam soluções explícitas para os estimadores EMV de $\beta$ e de $\Sigma_{\omega}$. Lindstrom and Bates (1988) examinam métodos para melhorar a eficiência computacional do algoritmo EM para esses modelos. Ware (1985) discute de modo geral algumas estruturas de covariância, incluindo o modelo multivariado geral, o modelo de efeitos aleatórios e o autoregressivo. Jennrich and Schluchter (1986) também discutem vários tipos de estruturas de covariância, incluindo modelos de efeitos aleatórios e AR(1), separadamente e usam diferentes algoritmos para obter as estimativas de máxima verossimilhança (Newton-Raphson, "scoring" de Fisher, EM, GEM). Covariáveis variando com o tempo e dados incompletos são também incorporadas. Schaalje et al. (1987) escreveram um programa (REMAC), que utiliza o procedimento de mínimos quadrados generalizados dos parâmetros $\beta \mathrm{em}$ (2.5.5) para as mesmas estruturas de covariância consideradas por Jennrich and Schluchter (1986) (exceto para o modelo de efeitos aleatórios). O programa REMAC não calcula as estimativas mais eficientes para os parâmetros, porém as estimativas obtidas são consistentes, simples e similares àquelas obtidas através da análise de modelos SPLIT-PLOT tradicional. Chi and Reinsel (1989) apresentam procedimentos para estimar os pararâmetros do modelo (2.5.5) com $\omega_{i j, k}=\phi \omega_{i j, k-1}+e_{i j, k}$, com $e_{i j, k} \sim \mathrm{N}\left(0, \sigma^{2}\right)$, que são similares ao método de MVR de Thompson (1969). 


\section{Capíitulo 3}

\section{Análise de Perfis para Experimentos em Blocos Aleatorizados}

\subsection{Introdução}

Grande parte dos experimentos com dados longitudinais têm como objetivo a comparação de diferentes tratamentos aplicados às unidades experimentais, o que, claramente, torna a homogeneidade uma característica desejável para os elementos da amostra a ser utilizada. No entanto, a realização de um experimento com unidades observacionais similares e em idênticas condições experimentais nem sempre é possível; em muitos casos há um ou mais fatores que, mesmo não sendo de maior interesse para 0 estudo, podem estar contribuindo para o aumento da variabilidade e devem, portanto, ser incorporados tanto no planejamento do experimento quanto na análise dos dados. Exemplos desse tipo de fatores foram vistos no Capítulo 1 e são baia (Exemplo 1.1), ninho (Exemplo 1.2) ou faixa de terra (Exemplo 1.3).

Uma saída para esse tipo de situação é a adoção de um planejamento em blocos, que consiste em dividir as unidades experimentais em grupos homogêneos, dentro dos quais elas sejam comparáveis, de modo a proporcionar uma redução nos erros experimentais.

Com base nas propostas de Andrade e Singer (1994), apresentamos neste Capítulo procedimentos para a análise de dados provenientes de estudos dessa natureza, ou seja, de dados gerados por planejamentos longitudinais em blocos. Analisamos dois casos: o primeiro, onde Blocos é considerado um fator fixo é tratado na Seção 3.2 e o segundo, onde se entende Blocos como um fator aleatório, na Seção 3.3. 


\subsection{Modelos de Efeitos Fixos para Blocos}

Vamos supor que o nosso conjunto de dados consiste de $p$ medidas seqüenciais realizadas em $n$ indivíduos divididos em $b$ blocos (considerados fixos), dentro dos quais foram aplicados $t$ tratamentos $(n=b t$, pois, por simplicidade, estamos assumindo um . planejamento em blocos completos).

No caso onde os dados são obtidos de um experimento em blocos completos aleatorizados, um enfoque comum é incluir blocos como um fator adicional (ver Milliken and Johnson (1984), por exemplo), gerando o modelo

$$
y_{i j k}=\alpha_{i k}+\mu_{j k}+\varepsilon_{i j k}
$$

$i=1, \ldots, b, j=1, \ldots, t, k=1, \ldots, p$ onde $\alpha_{i k}$ representa o efeito do $i$-ésimo bloco no $k$ ésimo instante de tempo, $\sum_{i=1}^{b} \alpha_{i k}=0, k=1, \mathrm{~K}, p, \quad \mu_{j k}$ representa a média do grupo submetido ao $j$-ésimo tratamento no $k$-ésimo instante de tempo e $\varepsilon_{i j k}$ é um erro com distribuição Normal e média zero. Este modelo pode ser representado na forma (2.3.1), $\operatorname{com} \boldsymbol{\beta}=\left(\mathbf{A}^{\mathrm{t}} \mathbf{M}^{\mathrm{t}}\right)^{\mathrm{t}}$ onde $\mathbf{A}=\left(\left(\alpha_{i k}\right)\right) \mathrm{e}$

$$
\mathbf{M}=\left[\begin{array}{cccc}
\mu_{11} & \mu_{12} & \cdots & \mu_{1 p} \\
\mu_{21} & \mu_{22} & \cdots & \mu_{2 p} \\
\vdots & \vdots & \ddots & \vdots \\
\mu_{t 1} & \mu_{t 2} & \cdots & \mu_{t p}
\end{array}\right] .
$$

Deste modo, qualquer hipótese linear que não envolva comparações de blocos pode ser expressa na forma $H: D \beta U=0$ com $D=[0 \mid C]$ onde $C$ é uma matriz de dimensão ( $c$ $\times t)$ e $\mathbf{0}$ é uma matriz nula de dimensão $(c \times b)$. Hipóteses que envolvem efeitos relacionados com blocos também podem ser consideradas, embora essa questão gere controvérsias (ver Samuels, Casella and McCabe (1991), por exemplo). A análise pode ser conduzida de maneira análoga àquela para experimentos completamente aleatorizados (ver Seção 2.3), o que torna esse enfoque bastante atraente.

Contudo, ele tem a desvantagem de requerer a inclusão da interação Blocos $\times$ Tempo no modelo. Apesar de existirem casos em que isso é conveniente (ver Steel and Torrie (1980), por exemplo), em muitas situações práticas (como em experimentos na 
área de Nutrição Animal) a inclusão de blocos é somente utilizada para controlar a homogeneidade das unidades experimentais e não se espera uma interação Bloco $\times$ Tempo.

Além disso, a inclusão dessa interação no modelo pode acarretar um aumento considerável $((b-1) t)$ no número de parâmetros. Caso $(b-1) t$ seja grande, muitos graus de liberdade estarão sendo utilizados para estimar parâmetros de pouca importância, ' diminuindo, assim, o poder dos testes relativos aos parâmetros que realmente nos interessam. Levando em conta esses argumentos, propomos um modelo sem interação Blocos $\times$ Tempo.

Nesse sentido, consideremos o seguinte modelo

$$
y_{i j k}=\alpha_{i}+\mu_{j k}+\varepsilon_{i j k}
$$

$\operatorname{com} \sum_{i=1}^{b} \alpha_{i}=0$, onde $\alpha_{i}$ representa o efeito do $i$-ésimo bloco.

Neste modelo são consideradas nulas as interações entre blocos e os demais fatores (Tratamento e Tempo). O número de parâmetros de localização passa a ser ( $b-1+$ $t p$ ), com uma redução de $(b-1) t$ parâmetros em relação ao modelo (3.2.1).

Para efeito de análise, a forma mais conveniente para representar o modelo (3.2.1) é

$$
y=\left(\mathbb{Z} \otimes 1_{p}\right) \alpha+\left[\left(1_{b} \otimes \mathbb{I}_{t}\right) \otimes \mathbb{I}_{p}\right] \mu+\varepsilon
$$

onde

$$
\begin{aligned}
& \underset{(b t p \times l)}{\boldsymbol{y}}=\overbrace{y_{111}, \ldots, y_{11 p}}^{\begin{array}{c}
\text { Bloco } 1 \\
\text { Tratamento } 1
\end{array}}, \overbrace{y_{121}, \ldots, y_{12 p}, \ldots, \overbrace{y_{1 t 1}, \ldots, y_{1 t p}}^{\text {Troatamento }}, \ldots, \overbrace{y_{b t 1}, \ldots, y_{b t p}}^{\text {Bloco } 1}}^{\mathrm{t}}, \\
& \underset{b t \times(b-1)}{\mathbf{Z}}=\left[\begin{array}{c}
\mathbf{I}_{b-1} \otimes \mathbb{1}_{t} \\
-\mathbf{1}_{b-1}^{t} \otimes \mathbb{1}_{t}
\end{array}\right], \\
& \alpha=\left(\alpha_{1}, \ldots, \alpha_{b-1}\right)^{t} \\
& \underset{(t p \times 1)}{\mu}=\left(\mu_{11}, \mu_{12}, \ldots, \mu_{1 p}, \mu_{21}, \ldots \mu_{2 p}, \ldots, \mu_{t 1}, \ldots \mu_{t p}\right)^{\mathrm{t}} .
\end{aligned}
$$

Aqui, o vetor $\varepsilon$ é dado por 


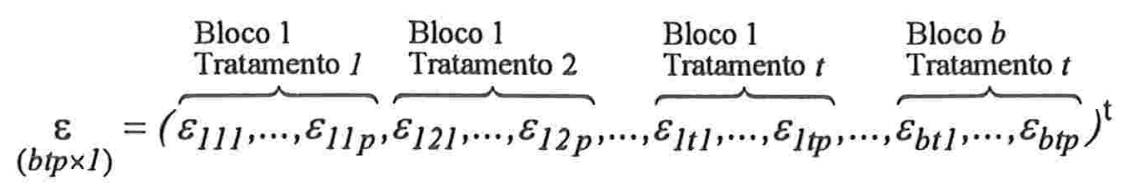

e $\operatorname{Var}(\varepsilon)=\mathbb{I}_{b t} \otimes \Sigma$, onde $\Sigma$ é uma matriz simétrica de posto $p$ positiva-definida e $\otimes$ denota o produto de Kronecker. Neste trabalho analisamos os casos em que $\Sigma$ tem estrutura uniforme ou é não-estruturada.

A formulação matricial acima é uma alternativa atraente devido à facilidade com que as hipóteses de interesse podem ser representadas na forma

$$
H: \mathbf{C} \mathbf{M} \mathbf{U}=\mathbf{0}
$$

onde C e U são matrizes de constantes conhecidas com dimensões $(c \times t)$ e $(p \times u)$ e postos $c$ e $u$, respectivamente. Na formulação vetorial (3.2.3) essa hipótese pode ser reexpressa como

$$
\begin{aligned}
H: & \left(\mathbf{C} \otimes \mathbf{U}^{t}\right) \mu=\mathbf{0} \Leftrightarrow \\
& \left(\mathbf{C} \otimes \mathbf{I}_{u}\right)\left(\mathbf{I}_{t} \otimes \mathbf{U}^{t}\right) \mu=\mathbf{0} \Leftrightarrow \\
& \left(\mathbf{C} \otimes \mathbf{I}_{u}\right) \mu^{*}=\mathbf{0}, \quad \operatorname{com} \quad \mu^{*}=\left(\mathbf{I}_{t} \otimes \mathbf{U}^{t}\right) \mu
\end{aligned}
$$

Esse fato sugere a seguinte transformação:

$$
\begin{aligned}
y^{*} & =\left(\mathbf{I}_{b t} \otimes \mathbf{U}^{t}\right) y \\
& \left.=\left(\mathbf{I}_{b t} \otimes \mathbf{U}^{t}\right)\left(\mathbf{Z} \otimes 1_{p}\right) \alpha+\left(\mathbf{I}_{b t} \otimes \mathbf{U}^{t}\right)\left[\left(\mathbf{1}_{b} \otimes \mathbf{I}_{t}\right) \otimes \mathbf{I}_{p}\right)\right] \mu+\left(\mathbf{I}_{b t} \otimes \mathbf{U}^{t}\right) \varepsilon \\
& \left.=\left(\mathbf{Z} \otimes \mathbf{U}^{t} 1_{p}\right) \alpha+\left[\mathbf{I}_{b t}\left(\mathbf{1}_{b} \otimes \mathbf{I}_{t}\right) \otimes \mathbf{U}^{t} \mathbf{I}_{p}\right)\right] \mu+\left(\mathbf{I}_{b t} \otimes \mathbf{U}^{t}\right) \varepsilon \\
& =\left(\mathbf{Z} \otimes \mathbf{U}^{t} 1_{p}\right) \alpha+\left[\left(\mathbf{1}_{b} \otimes \mathbf{I}_{t}\right) \otimes \mathbf{U}^{t}\right] \mu+\left(\mathbf{I}_{b t} \otimes \mathbf{U}^{t}\right) \varepsilon \\
& =\left(\mathbf{Z} \otimes \mathbf{U}^{t} 1_{p}\right) \alpha+\left[\left(\mathbf{1}_{b} \otimes \mathbf{I}_{t}\right) \otimes \mathbf{I}_{u}\right]\left[\mathbf{I}_{t} \otimes \mathbf{U}^{t}\right] \mu+\left(\mathbf{I}_{b t} \otimes \mathbf{U}^{t}\right) \varepsilon \\
& =\left(\mathbf{Z} \otimes \mathbf{U}^{t} 1_{p}\right) \alpha+\left(\mathbf{1}_{b} \otimes \mathbf{I}_{t u}\right) \mu^{*}+\varepsilon^{*} .
\end{aligned}
$$

Observe que se as colunas de $\mathbf{U}$ forem contrastes, como no caso dos testes de efeito de Tempo e de interação entre Tempo e Tratamento, $\mathbf{U}^{\mathrm{t}} \mathbf{1}_{p}=\mathbf{0}$ e a hipótese $H$ pode ser testada através do modelo

$$
y^{*}=\left(\mathbb{I}_{b} \otimes \mathbb{I}_{t u}\right) \mu^{*}+\varepsilon^{*}
$$


que corresponde essencialmente ao modelo usual para Análise de Perfis (os blocos são simplesmente tratados como repetições). Essa análise pode ser implementada de maneira análoga à apresentada nas Seções 2.3 (no caso em que nenhuma estrutura é imposta a $\Sigma$ ) ou 2.4 (no caso em que $\Sigma$ tem estrutura uniforme).

Por outro lado, se nem todas as colunas de $\mathbf{U}$ forem contrastes, é possível . distinguir dois casos:

a) U tem uma única coluna $(u=1)$, que não é um contraste. O modelo (3.2.7) reduz-se a

$$
y^{*}=\mathbf{Z}^{*} \alpha+\left(\mathbb{1}_{b} \otimes \mathbb{I}_{t u}\right) \mu^{*}+\varepsilon^{*}
$$

onde $\mathbb{Z}^{*}=k \mathbb{Z}$, com $k=\mathbb{U}^{t} \mathbf{1}_{p}$ e $\operatorname{Var}\left(\varepsilon^{*}\right)=\sigma^{2} \mathbb{I}_{b l}$, com $\sigma^{2}=\mathbf{U}^{t} \sum \mathbf{U}$. Esse modelo corresponde basicamente ao modelo empregado para a ANOVA em blocos. Essa análise pode ser implementada através de subrotinas para ANOVA, ANOVA com medidas repetidas ou MANOVA (que são equivalentes, neste caso), com os procedimentos GLM do SAS, 2V ou 4V do BMDP ou MANOVA/REPEATED do SPSS, por exemplo.

b) U tem duas ou mais colunas $(u \geq 2)$. Neste caso, não conhecemos soluções exatas; uma alternativa é utilizar testes de Wald baseados na distribuição assintótica dos estimadores de $\mathrm{MV}$, o que pode ser feito, em princípio, com o auxílio do módulo $5 \mathrm{~V}$ do programa BMDP, por exemplo (ver Apêndice A), ou considerar um procedimento em dois estágios em que as estimativas dos efeitos de blocos são obtidas através de a) com $\mathbf{U}=1_{p}$ e utilizadas para a obtenção de uma matriz de dados corrigida (com a eliminação do efeito de blocos), que então é analisada através de modelos usuais para Análise de Perfis. Nesse caso, é necessário corrigir o número de graus de liberdade do resíduo "entre" indivíduos através do fator $t p /\{t p-(b-1)\}$, descontando-se, assim, os graus de liberdade utilizados na estimação dos efeitos correspondentes a cada nível do fator Blocos. Essas duas últimas alternativas prestam-se, na realidade, para o teste de quaisquer hipóteses e não apenas aquelas para as quais posto($(\mathbb{U}) \geq 2$. 


\subsection{Modellos de Efeitos Aleatórios para Blocos}

Nesta seção abordaremos alguns procedimentos voltados para a análise de dados longitudinais gerados por planejamentos em blocos, agora tratando blocos como fatores aleatórios. Analogamente ao esquema adotado na Seção 3.2, descreveremos modelos que incluem a interação entre Blocos e Tempo e modelos sem essa interação.

Consideremos o seguinte modelo

$$
y=\left[\left(\mathbf{1}_{b} \otimes \mathbf{I}_{t}\right) \otimes \mathbb{I}_{p}\right] \mu+\varepsilon
$$

com $y=\left(y_{111}, y_{112}, \ldots, y_{b t p}\right)^{\mathrm{t}}$, onde $y_{i j k}$ é a observação no $i$-ésimo bloco para o $j$-ésimo tratamento na $k$-ésima condição de avaliação e $\varepsilon=\left(\varepsilon_{111}, \varepsilon_{112}, \ldots, \varepsilon_{b t p}\right)^{\imath}$ onde $\varepsilon_{i j k}$ é um erro aleatório que tem a forma

$$
\varepsilon_{i j k}=b_{i k}+\omega_{i j k}
$$

onde $b_{i k}$ e $\omega_{i j k}$ são variáveis aleatórias independentes e estão associadas ao bloco e ao indivíduo, respectivamente, no instante $k$. Para esses efeitos aleatórios são feitas as seguintes suposições:

$$
b_{i}=\left(b_{i 1}, \ldots, b_{i p}\right)^{t} \sim \mathrm{N}\left(0, \Sigma_{b}\right)
$$

e

$$
\omega_{i j}=\left(\omega_{i j 1}, \ldots, \omega_{i j p}\right)^{\iota} \sim \mathrm{N}\left(0, \Sigma_{\omega}\right)
$$

onde $\Sigma_{b}$ e $\Sigma_{\omega}$ são matrizes simétricas positivas definidas de dimensão $(p \times p)$.

Conseqüentemente,

$$
\varepsilon_{i}=\left(1_{i} \otimes b_{i}\right)+\omega_{i} \sim \operatorname{NID}\left(0, \Sigma_{\varepsilon}\right)
$$

e

$$
\Sigma_{\varepsilon}=\mathbf{I}_{t} \otimes \Sigma_{\omega}+\mathbf{1}_{t} \mathbf{1}_{t}^{\mathrm{t}} \otimes \Sigma_{b}
$$

Schaalje et al. (1991) propõem um procedimento em três etapas para o ajuste do modelo (3.3.1) no qual modelam diferentes componentes ortogonais que variam no 
tempo. Na primeira etapa, analisam-se as médias dos blocos ao longo do tempo $\left(\bar{y}_{i \bullet k}\right)$; na segunda analisam-se os desvios das médias dos blocos ao longo do tempo $\left(y_{i j k}-\bar{y}_{i \circ k}\right)$, enquanto que na terceira é feita uma combinação das informações obtidas nas duas primeiras etapas. Nas duas primeiras etapas, após o ajuste do modelo de médias aos dados usando o método dos Mínimos Quadrados Ordinários, calcula-se uma estimativa combinada da matriz de covariância utilizando os resíduos. Com base nessa estimativa da " matriz de covariância, o modelo de médias é reajustado através do procedimento de Mínimos Quadrados Generalizados. Uma segunda estimativa combinada da matriz de covariância é então obtida como anteriormente utilizando-se os resíduos do procedimento de Mínimos Quadrados Generalizados. A partir das estimativas dos parâmetros dessa matriz, diferentes estruturas para a matriz de covariância podem ser ajustadas. Para cada uma das matrizes de covariância estimadas, o procedimento de Mínimos Quadrados Generalizados é usado para, novamente, obterem-se estimativas dos parâmetros do modelo de médias. Na última etapa, os resultados das etapas anteriores são combinados para calcular as estatísticas de grau de ajuste do modelo, bem como estatísticas para se testarem hipóteses lineares (mais detalhes podem ser encontrados em Zhang et al., 1987).

O procedimento acima foi implementado pelos próprios autores utilizando a linguagem IML do programa SAS. Esse enfoque não será considerado com mais detalhes neste projeto.

Retirando-se a interação aleatória entre Blocos e Tempo do modelo (3.3.1), ou seja, substituindo-se (3.3.2) por

$$
\varepsilon_{i j k}=b_{i}+\omega_{i j k}
$$

onde $b_{i} \sim \mathrm{N}\left(0, \sigma_{b}^{2}\right)$, e mantendo as demais suposições, obtemos

$$
\operatorname{Var}(\varepsilon)=\mathbf{I}_{b} \otimes\left(\sigma_{b}^{2} \mathbf{1}_{t p} \mathbf{1}_{t p}^{\mathrm{t}}+\mathbf{I}_{t} \otimes \Sigma_{w}\right) .
$$

Note que $\operatorname{Var}\left(\varepsilon_{i j}\right)=\operatorname{Var}\left(y_{i j}\right)$ não satisfaz às condições necessárias para a aplicação dos métodos considerados nas Seções 2.3 ou 2.4 .

Contudo, uma transformação como aquela considerada na Seção 3.3 reduz (3.3.1) a 


$$
\begin{aligned}
y^{*} & =\left(\mathbb{I}_{b t} \otimes \mathbf{U}^{t}\right) \\
& =\left(\mathbb{I}_{b t} \otimes \mathbf{U}^{t}\right)\left\{\left[\left(\mathbb{1}_{b} \otimes \mathbb{I}_{t}\right) \otimes \mathbb{I}_{p}\right] \mu+\varepsilon\right\} \\
& =\left(\mathbb{1}_{b} \otimes \mathbf{I}_{t u}\right) \mu^{*}+\varepsilon^{*}
\end{aligned}
$$

onde $\varepsilon^{*}=\left(\mathbf{I}_{b t} \otimes \mathbf{U}^{t}\right) \varepsilon$ tem matriz de covariância

$$
\operatorname{Var}\left(\varepsilon^{*}\right)=\mathbf{I}_{b} \otimes\left\{\sigma_{b}^{2}\left(\mathbf{1}_{t}^{\mathbf{t}_{t}^{\mathrm{t}}} \otimes \mathbf{U}^{\mathrm{t}} \mathbf{1}_{p}^{\mathbf{1}_{p}^{\mathrm{t}}}\right)+\mathbf{I}_{t} \otimes \mathbf{U}^{\mathrm{t}} \Sigma \mathbf{U}\right\}
$$

Como na Seção 3.1, a decisão quanto à estratégia de análise está vinculada ao tipo das matrizes $\mathbf{U}$ que definem as hipóteses, destacando que somente a estrutura de covariância é afetada. Consideremos as mesmas três alternativas.

a) Todas as colunas de U são contrastes.

Então (3.3.8) reduz -se a

$$
\operatorname{Var}(\varepsilon)=\mathbf{I}_{b t} \otimes \mathbf{U}^{t} \Sigma \mathbf{U}
$$

que tem estrutura uniforme ou é não-estruturada, dependendo de $\Sigma$ ser uniforme ou ser não-estruturada. As hipóteses da forma $\mathrm{H}$ : $\mathbf{C M U}=\mathbf{0}$ podem ser testadas através dos mesmos modelos utilizados no caso de blocos fixos (com blocos considerados como réplicas).

b) U tem uma única coluna que não é de contrastes.

Neste caso (3.3.8) reduz-se a

$$
\operatorname{Var}\left(\varepsilon^{*}\right)=\mathbf{I}_{b} \otimes\left(\sigma_{b U}^{2} \mathbf{1}_{t} \mathbf{1}_{t}^{t}+\sigma_{U}^{2} \mathbf{I}_{t}\right)
$$

onde $\sigma_{b U}^{2}=\sigma_{b}^{2}\left(\mathbf{U}^{t} \mathbf{1}_{p}\right)^{2}$ e $\sigma_{U}^{2}=\mathbf{U}^{t} \Sigma \mathbf{U}$. Portanto as hipóteses de interesse podem ser testadas via modelos ANOVA com blocos aleatórios.

c) U tem mais de uma coluna e nem todas são contrastes.

Neste caso, a transformação sugerida não reduz $\operatorname{Var}\left(\varepsilon^{*}\right)$ a uma forma que permita a análise através dos métodos considerados nas Seções 2.3 e 2.4 e as hipóteses correspondentes podem ser testadas via métodos de máxima verossimilhança. 


\section{Capítulo 4}

\section{Exemplo Numérico}

A fim de ilustrarmos os métodos propostos no Capítulo 3, apresentamos, neste capítulo, os resultados da análise do conjunto de dados do Exemplo 1.1

\subsection{Análise dos dados do Exemplo 1.1}

Como já foi descrito no Capítulo 1, trata-se de um experimento conduzido com 5 Tratamentos, 4 Blocos e 4 medidas semanais. Apresentamos nesta seção, apenas para efeitos ilustrativos, os resultados obtidos ao analisarmos os dados utilizando as várias modelagens propostas (com o auxílio do programa SAS/GLM). Dado que os pesos iniciais dos animais foram utilizados para definir os blocos, não incluímos essa medida na análise. Inicialmente, consideramos Blocos como um fator fixo.

Ajustamos em primeiro lugar o modelo (3.2.1) incluindo os efeitos de Blocos, Tratamento, Tempo, interação Tratamento $\times$ Tempo e interação Blocos $\times$ Tempo (modelo (3.2.1)). Na Tabela 4.1.1 estão os resultados dos testes padrão de uma Análise de Perfis com a matriz de covariâncias $\Sigma$ não estruturada. Em geral, hipóteses relacionadas com efeitos de Blocos não têm interesse prático e testes dessas hipóteses podem gerar controvérsias; apesar disso, nós consideramos esses testes aqui somente como uma forma de descrever uma situação onde a interação Blocos $\times$ Tempo não deveria ser incluída no modelo. 
Tabela 4.1.1. Análise de Perfis para os dados correspondentes ao Exemplo 1.1 ( $\Sigma$ não estruturada ).

\begin{tabular}{|c|c|c|c|c|}
\hline $\begin{array}{l}\text { Hipótese de } \\
\text { inexistência do efeito }\end{array}$ & $\begin{array}{c}\text { Estatística do } \\
\text { teste * }\end{array}$ & $\mathrm{F}$ & GL & $\begin{array}{c}\text { Nível } \\
\text { descritivo }\end{array}$ \\
\hline \multirow{4}{*}{ Tratamento $\times$ Tempo } & $P=0,7031$ & 1,6264 & $8 ; 24$ & 0,1695 \\
\hline & $H=1,2041$ & 1,5051 & $8 ; 20$ & 0,2173 \\
\hline & $W=0,4048$ & 1,5051 & $8 ; 22$ & 0,1901 \\
\hline & $R=0,9118$ & 2,7354 & $4 ; 12$ & 0,0791 \\
\hline \multirow{4}{*}{ Blocos $\times$ Tempo } & $P=0,5985$ & 1,7083 & $6 ; 24$ & 0,1623 \\
\hline & $H=1,2393$ & 2,0654 & $6 ; 20$ & 0,1037 \\
\hline & $W=0,4326$ & 1,9078 & $6 ; 22$ & 0,1246 \\
\hline & $R=1,1781$ & 4,7123 & $3 ; 12$ & 0,0214 \\
\hline \multirow{4}{*}{ Tempo } & $P=0,9975$ & 2211,01 & $2 ; 11$ & $<0,0001$ \\
\hline & $H=402,0014$ & 2211,01 & $2 ; 11$ & $<0,0001$ \\
\hline & $W=0,0025$ & 2211,01 & $2 ; 11$ & $<0,0001$ \\
\hline & $R=402,0014$ & & $2 ; 11$ & $<0,0001$ \\
\hline Tratamento & $\mathrm{F}=3,05$ & & $4 ; 12$ & 0,0598 \\
\hline Blocos & $F=11,50$ & & $3 ; 12$ & 0,0008 \\
\hline
\end{tabular}

* $P=$ Pillai, $H=$ Lawley-Hotelling, $W=$ Wilks, $R=$ Roy

Utilizando o critério de Mauchly (1940), a hipótese de circularidade para a matriz $\Sigma$ (ver condição (2.4.5)) não deve ser rejeitada $(\mathrm{p}=0,8243)$, indicando que o modelo misto (2.4.1) poderia ser adotado neste caso.

Na Tabela 4.1.2 apresentamos os resultados da mesma Análise de Perfis ("intra" indivíduos) através do modelo misto (em que $\Sigma$ tem estrutura uniforme). Não incluímos os testes referentes à inexistência de efeitos de Tratamento e Blocos porque são análogos aos obtidos quando $\Sigma$ é não estruturada. 
Tabela 4.1.2. Análise de Perfis para os dados do Exemplo (1.1) segundo o Modelo Misto Univariado ( $\Sigma$ uniforme)

\begin{tabular}{|c|c|c|c|}
\hline $\begin{array}{l}\text { Hipótese de inexistência } \\
\text { do efeito de }\end{array}$ & $\mathrm{F}$ & G.L. & $\begin{array}{c}\text { Nível } \\
\text { Descritivo* }\end{array}$ \\
\hline Tratamento $\times$ Tempo & 1,97 & $8 ; 24$ & $\begin{aligned} p & =0,0948 \\
\mathrm{G}-\mathrm{G} & =0,0982 \\
\mathrm{H}-\mathrm{F} & =0,0948\end{aligned}$ \\
\hline Blocos $\times$ Tempo & 2,81 & $6 ; 24$ & $\begin{aligned} p & =0,0324 \\
G-G & =0,0344 \\
H-F & =0,0324\end{aligned}$ \\
\hline Tempo & 2641,59 & $2 ; 24$ & $\begin{array}{l}\mathrm{p}=0,0001 \\
\mathrm{G}-\mathrm{G}<0,0001 \\
\mathrm{H}-\mathrm{F}<0,0001\end{array}$ \\
\hline
\end{tabular}

* $\mathrm{p}=$ não corrigido, G-G = com correção de Greenhouse-Geisser, $\mathrm{H}-\mathrm{F}=$ com correção de Huynh e Feldt

Note que nesse caso há uma indicação de uma possível interação Tratamento $\times$ Tempo ( $p<0,0982)$, que não é tão evidente com os testes multivariados ( $p>0,1695)$, com exceção do teste de Roy, para o qual $p=0,0791$.

Embora a interação Blocos $\times$ Tempo seja significativa neste caso ( $p<0,0344)$, os resultados obtidos através do modelo multivariado ( $p>0.10$, com exceção do teste de Roy, para o qual $\mathrm{p}=0,0214$ ), aliados à justificativa apresentada anteriormente, sugerem que o modelo sem essa interação (ver 3.3.1) poderia ser uma alternativa para a análise dos dados em questão. A idéia de que a interação entre Tempo e Blocos pode ser desprezada ${ }^{\prime}$ reforçada pela Figura 4.1.1, onde se observa um paralelismo entre os perfis de resposta dos blocos. 
Figura 4.1.1 Perfis médios de resposta dos Blocos para cada condição de avaliação.

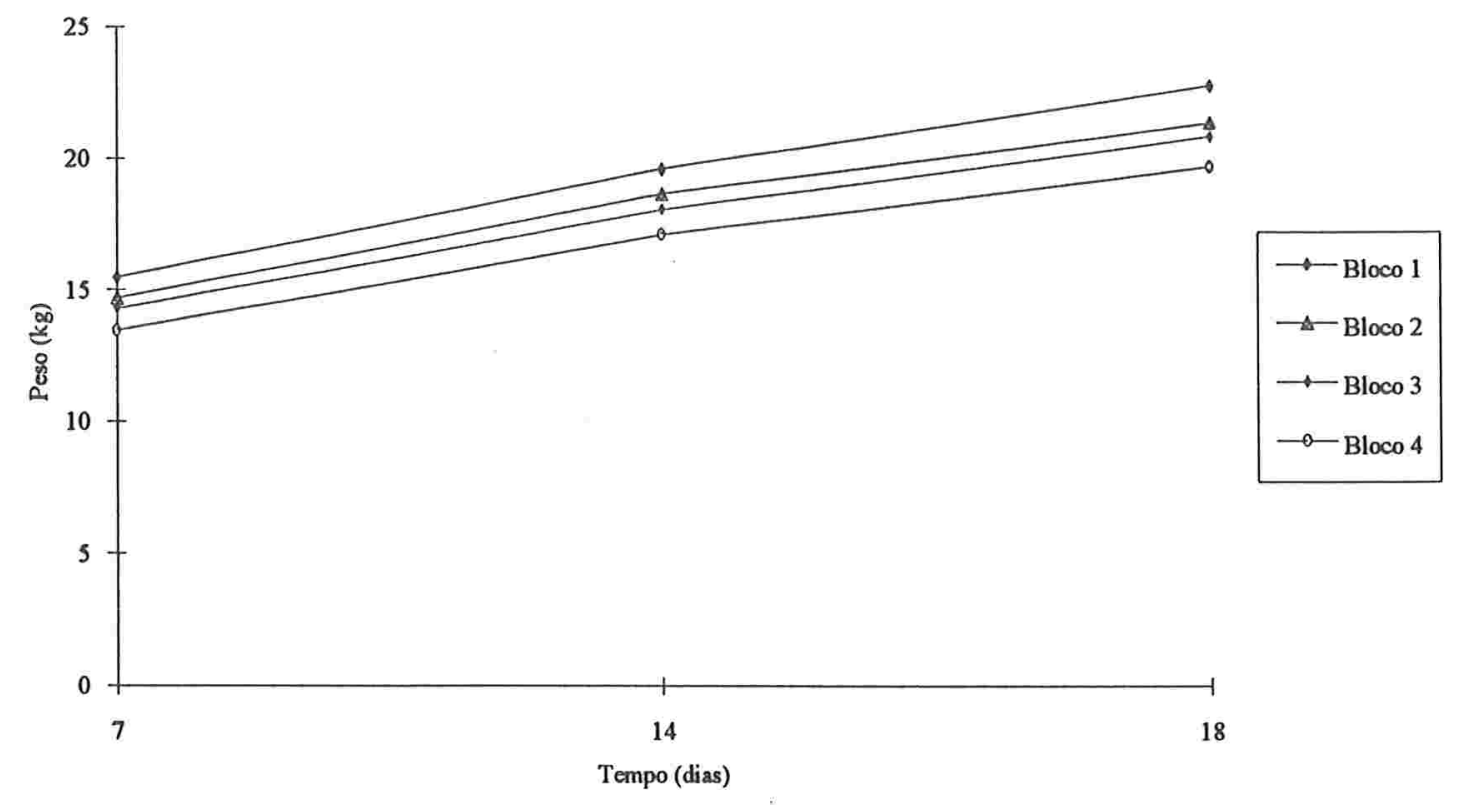

Sob esse modelo, as hipóteses de inexistência de interação entre Tratamento e Tempo e de inexistência de efeito de Tempo, são tais que

$$
\mathbf{U}=\left[\begin{array}{cccc}
1 & 1 & 1 & 1 \\
-1 & 0 & 0 & 0 \\
0 & -1 & 0 & 0 \\
0 & 0 & -1 & 0 \\
0 & 0 & 0 & -1
\end{array}\right]
$$

Como nesse caso as colunas da matriz U são contrastes, essas hipóteses foram testadas considerando-se blocos como repetições. Os resultados estão expressos nas Tabelas 4.1.3 e 4.1.4. 
Tabela 4.1.3. Análise de Perfis para os dados do Exemplo 1.1 com $\Sigma$ não estruturada, considerando Blocos como réplicas

\begin{tabular}{lcccc}
\hline $\begin{array}{l}\text { Hipótese de inexistência } \\
\text { do efeito de }\end{array}$ & $\begin{array}{c}\text { Estatística do } \\
\text { teste } *\end{array}$ & F & GL & $\begin{array}{c}\text { Nível } \\
\text { descritivo }\end{array}$ \\
\hline Tratamento $\times$ Tempo & $\begin{array}{l}P=0,5136 \\
H=0,7036\end{array}$ & 1,30 & $8 ; 30$ & 0,2830 \\
$(\mathrm{~s}=2, \mathrm{v}=0.5, \mathrm{t}=6)$ & $W=0,5498$ & 1,22 & $8 ; 26$ & 0,3688 \\
& $R=0,4439$ & 1,66 & $4 ; 15$ & 0,3236 \\
& & & & \\
\hline & $P=0,9948$ & 1344,100 & $2 ; 14$ & 0,0001 \\
Tempo & $H=192,0142$ & 1344,100 & $2 ; 14$ & 0,0001 \\
$(\mathrm{~s}=1, \mathrm{v}=0, \mathrm{t}=6)$ & $W=0,0052$ & 1344,100 & $2 ; 14$ & 0,0001 \\
& $R=192,0142$ & 1344,100 & $2 ; 14$ & 0,0001 \\
\hline
\end{tabular}

* $P=$ Pillai, $H=$ Lawley-Hotelling, $W=$ Wilks, $R=$ Roy

Tabela 4.1.4. Análise dos dados do Exemplo 1.1 segundo o Modelo Misto Univariado ( $\Sigma$ uniforme) onde Blocos são tratados como réplicas

\begin{tabular}{|c|c|c|c|}
\hline $\begin{array}{l}\text { Hipótese de inexistência } \\
\text { do efeito de }\end{array}$ & F & GL & $\begin{array}{r}\text { Nível } \\
\text { descritivo* }^{*}\end{array}$ \\
\hline Tratamento $\times$ Tempo & 1,45 & $8 ; 30$ & $\begin{array}{l}\mathrm{p}=0,2180 \\
\mathrm{G}-\mathrm{G}=0,2348 \\
\mathrm{H}-\mathrm{F}=0,2180\end{array}$ \\
\hline Tempo & 1939,01 & $2 ; 30$ & $\begin{array}{cr}\mathrm{p} & 0,0001 \\
\mathrm{G}-\mathrm{G}= & 0,0001 \\
\mathrm{H}-\mathrm{F}= & 0,0001\end{array}$ \\
\hline
\end{tabular}

Note que os resultados das Tabelas 4.1.3 e 4.1.4 sugerem a inexistência da interação Tratamento $\times$ Tempo $(p>0,2102$ e $p>0,2180$, respectivamente), confirmando os resultados dos testes multivariados da Tabela 4.1.1. Neste caso, para o teste de Roy, $p$ $=0,2102$, o que o torna compatível com os demais testes multivariados. 
A hipótese de inexistência de efeito de Tratamento foi testada através de um modelo univariado em blocos, ajustado às somas das observações nas 3 ocasiões em que as unidades experimentais foram avaliadas (ver item (a) da Seção 3.3) tendo-se obtido $\mathrm{p}=0,0598$, valor que naturalmente coincide com o teste correspondente na Tabela 4.1.1.

Os testes de efeito de Tempo e interação Tratamento $\times$ Tempo na Tabela (4.1.3) foram realizados com o auxílio do módulo $4 \mathrm{~V}$ do programa BMDP. Este mesmo programa permite-nos testar a hipótese de inexistência de efeito de Tratamento através de um modelo univariado em blocos, utilizando a sugestão apresentada em (3.3.7).

Como foi mencionados no Capítulo 3, tanto o método da máxima verossimilhança como o procedimento em dois estágios podem ser usados em todos os casos, independentemente da forma da matriz U. Ilustrando o procedimento em dois estágios, testamos inicialmente as hipóteses padrão. Para este conjunto de dados, as estimativas dos efeitos de blocos são $\hat{\alpha}_{1}=1,2845, \hat{\alpha}_{2}=0,23458, \hat{\alpha}_{3}=-0,27042, \hat{\alpha}_{4}=-1,2487$. Os resíduos foram calculados extraindo-se de cada observação a estimativa do efeito correspondente ao bloco a que pertenciam. Na Tabela 4.1.5 encontram-se as médias e os desvios padrões estimados por Tratamento e Tempo dos dados originais e dos resíduos. 
Tabela 4.1.5. Estimativas das médias dos pesos dos leitões e dos respectivos desvios padrões (Exemplo 1.1)

\begin{tabular}{|c|c|c|c|c|}
\hline & \multicolumn{4}{|c|}{ Peso } \\
\hline & Tratamento & 7 dias & 14 dias & 18 dias \\
\hline \multirow{5}{*}{$\begin{array}{l}\text { Dados } \\
\text { Originais }\end{array}$} & 1 & $\begin{array}{r}14,987 \\
(0,942)\end{array}$ & $\begin{array}{r}19,162 \\
(1,167)\end{array}$ & $\begin{array}{r}22,025 \\
(1,236)\end{array}$ \\
\hline & 2 & $\begin{array}{r}14,687 \\
(0,918)\end{array}$ & $\begin{array}{r}18,512 \\
(0,9420)\end{array}$ & $\begin{array}{r}21,719 \\
(1,258)\end{array}$ \\
\hline & 3 & $\begin{array}{r}13,800 \\
(1,009)\end{array}$ & $\begin{array}{r}17,462 \\
(1,342)\end{array}$ & $\begin{array}{r}19,987 \\
(1,604)\end{array}$ \\
\hline & 4 & $\begin{array}{r}14,381 \\
(0,997)\end{array}$ & $\begin{array}{r}18,356 \\
(1,337)\end{array}$ & $\begin{array}{r}20,881 \\
(1,436)\end{array}$ \\
\hline & 5 & $\begin{array}{r}14,531 \\
(0,998)\end{array}$ & $\begin{array}{r}18,319 \\
(1,497)\end{array}$ & $\begin{array}{r}21,219 \\
(1,760)\end{array}$ \\
\hline \multirow{5}{*}{ Resíduos } & 1 & $\begin{array}{r}14,987 \\
(0,245)\end{array}$ & $\begin{array}{r}19,162 \\
(0,351)\end{array}$ & $\begin{array}{r}22,025 \\
(0,331)\end{array}$ \\
\hline & 2 & $\begin{array}{r}14,6875 \\
(0,743)\end{array}$ & $\begin{array}{r}18,512 \\
(0,986)\end{array}$ & $\begin{array}{r}21,719 \\
(0,765)\end{array}$ \\
\hline & 3 & $\begin{array}{r}13,800 \\
(0,505)\end{array}$ & $\begin{array}{r}17,462 \\
(0,771)\end{array}$ & $\begin{array}{r}19,987 \\
(1,028)\end{array}$ \\
\hline & 4 & $\begin{array}{r}14,381 \\
(0,469)\end{array}$ & $\begin{array}{r}18,356 \\
(0,635)\end{array}$ & $\begin{array}{r}20,881 \\
(0,756)\end{array}$ \\
\hline & 5 & $\begin{array}{r}14,531 \\
(0,482)\end{array}$ & $\begin{array}{r}18,319 \\
(0,740)\end{array}$ & $\begin{array}{l}21,219 \\
(0,827)\end{array}$ \\
\hline
\end{tabular}

Observando-se a Tabela 4.1.5, nota-se que as médias dos tratamentos permaneceram inalteradas ao se extraírem as estimativas dos efeitos dos Blocos, diferentemente dos desvios padrões, que sofreram uma diminuição considerável.

Os resultados de uma Análise de Perfis tendo os resíduos como variáveis resposta estão expressos na Tabela 4.1.6. 
Tabela 4.1.6. Análise de Perfis para os resíduos correspondentes ao Exemplo 1.1 ( $\Sigma$ não estruturada.)

\begin{tabular}{|c|c|c|c|c|}
\hline $\begin{array}{l}\text { Hipótese de } \\
\text { inexistência do efeito }\end{array}$ & $\begin{array}{c}\text { Estatística do } \\
\text { teste } *\end{array}$ & $\mathrm{~F}$ & G.L. & $\begin{array}{c}\text { Nível } \\
\text { descritivo }\end{array}$ \\
\hline \multirow{4}{*}{$\begin{array}{l}\text { Tratamento } \times \text { Tempo } \\
(\mathrm{s}=2, \mathrm{v}=0.5, \mathrm{t}=6)\end{array}$} & $P=0,5136$ & 1,30 & $8 ; 30$ & 0,2830 \\
\hline & $H=0,7036$ & 1,14 & $8 ; 26$ & 0,3688 \\
\hline & $W=0,5498$ & 1,22 & $8 ; 28$ & 0,3236 \\
\hline & $R=0,4439$ & 1,66 & $4 ; 15$ & 0,2102 \\
\hline \multirow{4}{*}{$\begin{array}{l}\text { Tempo } \\
(s=1, v=0, t=6)\end{array}$} & $P=0,9948$ & 1344,100 & $2 ; 14$ & 0,0001 \\
\hline & $H=192,0142$ & 1344,100 & $2 ; 14$ & 0,0001 \\
\hline & $W=\quad 0,0052$ & 1344,100 & $2 ; 14$ & 0,0001 \\
\hline & $R=192,0142$ & 1344,100 & $2 ; 14$ & 0,0001 \\
\hline Tratamento & $F=3,81$ & & $4 ; 15$ & 0,0248 \\
\hline
\end{tabular}

* $P=$ Pillai, $H=$ Lawley-Hotelling, $W=$ Wilks, $R=$ Roy

Como se pode observar, os resultados dos testes para os efeitos de Tempo e de interação Tratamento $\times$ Tempo na Tabela 4.1.5 são análogos aos obtidos na Tabela 4.1.3. $\mathrm{O}$ mesmo ocorre quando $\Sigma$ tem estrutura uniforme.

Neste caso, é necessário corrigir os graus de liberdade das estatísticas de teste, descontando aqueles usados para se estimar o efeito de Blocos. A saída do programa fornece o valor 1,1613 com 15 graus de liberdade para o Quadrado Médio do Resíduo. Como temos 4 blocos, o número de graus de liberdade associado ao resíduo "entre" indivíduos é, na realidade, 12; portanto

$$
\text { QMR corrigido }=1,1613 \times 15 / 12=1,4516
$$

O Quadrado Médio associado a Tratamento é 4,4290; obtém-se, assim, para o teste referente ao efeito de Tratamento, a estatística $F=3,05$, com 4 e 12 graus de liberdade no numerador e no denominador, respectivamente $(p=0,0598)$. Este valor coincide com aqueles obtidos na Tabela 4.1.1 e também com aqueles obtidos através do modelo univariado em blocos. 
Verifiquemos agora o caso onde a matriz $\mathbf{U}$ tem posto $\geq 2$ e não é uma matriz de contrastes. Testemos, por exemplo a hipótese conjunta de que tanto a resposta média no primeiro instante de observação, quanto a média dos instantes 2 e 3 são iguais para os tratamentos 1 e $3\left(H_{1}\right)$. Analogamente, testemos a hipótese conjunta de que tanto a resposta média no primeiro instante de observação quanto a média dos instantes 2 e 3 são iguais para os cinco tratamentos $\left(H_{2}\right)$. Para esta situação,

$$
\mathbf{U}_{1}=\left[\begin{array}{ll}
1 & 0 \\
0 & 1 \\
0 & 1
\end{array}\right]
$$
hipóteses

Trabalhando com os resíduos (observações - efeito de bloco), testamos as

i) $H_{1}: \mathbf{C}_{1} \mathbf{M U}_{1}=0, \operatorname{com} \mathbf{C}_{1}=\left[\begin{array}{lllll}1 & 0 & -1 & 0 & 0\end{array}\right]$

e

ii) $H_{2}: \mathbf{C}_{2} \mathbf{M U}_{1}=\mathbf{0}$, com

$$
\mathrm{C}_{2}=\left[\begin{array}{ccccc}
1 & -1 & 0 & 0 & 0 \\
1 & 0 & -1 & 0 & 0 \\
1 & 0 & 0 & -1 & 0 \\
1 & 0 & 0 & 0 & -1
\end{array}\right]
$$

Aqui também, os graus de liberdade das estatísticas de teste devem ser corrigidos para levar em conta a estimação do efeito de Blocos. Para a hipótese $H_{1}$, a estatística $\mathrm{F}$ obtida da saída do programa BMDP/4V tem 2 graus de liberdade no numerador e $2 *(20-5)=30$ graus de liberdade no denominador. Com a correção, F passa a ser 10.28 e os graus de liberdade do denominador passam a ser $2 *(20-5-3)=24$. Nesse caso, os níveis descritivos correspondentes passam a ser $\mathrm{p}=0,0165, \mathrm{G}-\mathrm{G}=0,0059$ e $\mathrm{H}-\mathrm{F}=0,0042$. Quanto à hipótese $\mathrm{H}_{2}$, os graus de liberdade do numerador e do denominador da estatística $\mathrm{F}$ fornecida pelo $\mathrm{BMDP} / 4 \mathrm{~V}$ são, respectivamente, $2 * 4=8$ e $2 *(20-5)=30$. Este último também sofre correção e passa a ser 24 , obtendo-se então $F=3.04$ e os níveis descritivos $p=0,0165$, $\mathrm{G}-\mathrm{G}=0,0432$ e $\mathrm{H}-\mathrm{F}=0,0226$. 
A Tabela seguinte contém os resultados obtidos através dos testes de Wald para as hipóteses de inexistência de efeito de Blocos, Tratamento, Tempo e interação Tratamento $\times$ Tempo referentes ao modelo (3.2.1) para $\Sigma$ com as duas estruturas discutidas acima.

Tabela 4.1.7. Teste de Wald para os efeitos fixos do modelo 3.2.1

\begin{tabular}{|c|c|c|c|c|}
\hline Padrão para $\Sigma$ & $\begin{array}{l}\text { Teste de inexistência } \\
\text { de efeito de }\end{array}$ & G.L. & $\chi^{2}$ & $\begin{array}{r}\text { Nível } \\
\text { descritivo }\end{array}$ \\
\hline \multirow{5}{*}{ Uniforme } & Blocos & 3 & 34,49 & $<0,001$ \\
\hline & Tratamento & 4 & 12,20 & 0,016 \\
\hline & Tempo & 2 & 5281,69 & $<0,001$ \\
\hline & Blocos $\times$ Tempo & 6 & 16,87 & 0,010 \\
\hline & Tratamento $\times$ Tempo & 8 & 15,77 & 0,046 \\
\hline \multirow{5}{*}{ Não Estruturada } & Blocos & 3 & 33,87 & $<0,001$ \\
\hline & Tratamento & 4 & 9,43 & 0,016 \\
\hline & Tempo & 2 & 4823,50 & $<0,001$ \\
\hline & Blocos $\times$ Tempo & 6 & 14,87 & 0,021 \\
\hline & Tratamento $\times$ Tempo & 8 & 14,45 & 0,071 \\
\hline
\end{tabular}

Retirando-se o efeito de interação Blocos $\times$ Tratamento do modelo (modelo 3.3.1) obtêm-se os resultados apresentados na Tabela 4.1.8.

Tabela 4.1.8. Testes de Wald para os efeitos fixos do modelo 3.3.1.

\begin{tabular}{llrrr}
\hline Padrão para $\Sigma$ & $\begin{array}{c}\text { Teste de inexistência } \\
\text { do efeito }\end{array}$ & G.L. & $\chi^{2}$ & $\begin{array}{r}\text { Nível } \\
\text { descritivo }\end{array}$ \\
\hline \multirow{3}{*}{ Uniforme } & Blocos & 3 & 34,47 & $<0,001$ \\
& Tratamento & 4 & 12,20 & 0,016 \\
& Tempo & 2 & 3877,73 & $<0,001$ \\
& Tratamento $\times$ Tempo & 8 & 11,58 & 0,171 \\
\hline \multirow{3}{*}{ Não Estruturada a } & Tratamento & 3 & 33,87 & $<0,001$ \\
& Tempo & 4 & 9,43 & 0,051 \\
& Tratamento $\times$ Tempo & 8 & 10,55 & 0,228 \\
\hline
\end{tabular}


Os resultados fornecidos pelo Teste de Wald vêm confirmar as conclusões obtidas anteriormente para esse conjunto de dados; em particular, a interação Tratamento $\times$ Tempo, que era significativa $(p=0,046$ para $\Sigma$ uniforme e $p=0,071$ para $\Sigma$ não estruturada) com a inclusão da interação Blocos $\times$ Tempo, passa a ser não-significativa ( $p$ $=0,171$ para $\Sigma$ uniforme e $\mathrm{p}=0,228$ para $\Sigma$ não estruturada) quando essa interação é ' retirada do modelo.

Em versões anteriores do programa $\mathbf{B M D P} / 5 \mathrm{~V}$, o teste de Wald só testa a significância dos efeitos do modelo, mas na versão 7.0 há um novo comando, ESTIMATE, que permite testar outras hipóteses, inclusive aquelas para as quais posto(U) $\geq 2$. Para tanto, é necessário representar cada média de casela como uma combinação linear de parâmetros de regressão e formular as hipóteses de interesse. Como ainda não dispomos dessa nova versão, deixamos de apresentar os resultados dos testes de Wald para outras hipóteses.

Caso desejássemos considerar Blocos como um fator aleatório, de acordo com os procedimentos descritos na Seção 3.3, os resultados dos testes de inexistência de efeito de Tempo e de interação Tempo $\times$ Tratamento seriam os mesmos que os das Tabelas 4.1.3 ou 4.1.4, uma vez que nestes casos o efeito de Blocos se anula. Já a hipótese de inexistência de efeito de Tratamento seria testada utilizando-se um modelo univariado com blocos aleatórios, o que pode ser feito com o auxílio dos programas BMDP/3V ou BMDP//8V. Neste caso, obter-se-ia $F=3,05$ com 4 e 12 graus de liberdade no numerador e denominador, respectivamente, $(p=0,0598)$, que coincide com o resultado encontrado no caso de blocos fixos. 


\subsection{Considerações Finais}

No que se refere a experimentos com planejamentos em blocos, procuramos nos concentrar, neste trabalho, nas técnicas voltadas para $\Sigma$ não estruturada ou com estrutura uniforme, que são as mais utilizadas e de mais fácil implementação. Vimos no Capítulo 2 ' outras alternativas, talvez até mais adequadas para explicar a estrutura de covariância "intra" indivíduo. Sugerimos, para trabalhos futuros nesta área, que se estude a implementação de outras estruturas como $\mathrm{AR}(1)$ no caso de dados longitudinais com planejamento em blocos. 


\section{Apêndice A}

\section{Estimação e Testes - Método da Máxima Verossimillhança}

Sob a suposição

$$
y_{i j}=\left(y_{i j 1}, y_{i j 2}, \ldots, y_{i j p}\right)^{\mathrm{t}} \sim N_{p}\left(\mathbb{Z}_{i j} \alpha+\mathbf{X}_{i j} \mu, \Sigma_{i j}\right),
$$

a função de verossimilhança associada ao modelo (3.2.2), que denotaremos por $\lambda$, é dada por

$$
\lambda=(2 \pi)^{-n / 2} \prod_{i=1}^{b} \prod_{j=1}^{t}\left|\Sigma_{i j}\right|^{-1 / 2} \exp \left[-\frac{1}{2} \sum_{i=1}^{b} \sum_{j=1}^{t}\left(y_{i j}-\mathrm{W}_{i j} \eta\right) \Sigma_{i j}^{-1}\left(y_{i j}-\mathrm{W}_{i j} \eta\right)\right]
$$

Além disso, $\quad \sum_{i j}=\Sigma_{i j}(\theta)$, ou seja, a matriz de covariâncias pode ser escrita como função de um determinado vetor de parâmetros e adotamos a notação $\mathbf{W}_{i}=\left[\mathbb{Z}_{i} \mid \mathbf{X}_{i}\right], \eta=\left[\alpha^{t} \mu^{t}\right]^{t}$.

Para obtermos estimativas de máxima verossimilhança de $\eta$ e $\theta$, precisamos encontrar $\hat{\eta}$ e $\hat{\theta}$ que maximizam (A.1), o que é equivalente a maximizar o logaritmo da função de verossimilhança

$$
\ln \lambda=K-\frac{1}{2} \sum_{i=1}^{b} \sum_{j=1}^{t} \ln \left|\Sigma_{i j}\right|-\frac{1}{2} \sum_{i=1}^{b} \sum_{j=1}^{t}\left(y_{i j}-W_{i j} \eta\right) \Sigma_{i j}^{-1}\left(y_{i j}-W_{i j} \eta\right)
$$


onde $K$ é uma constante que não depende de $\eta$ nem $\theta$. Necessitamos, pois, calcular as primeiras e segundas derivadas parciais de $\log \lambda$ em relação a $\eta$ e $\theta$. Essas derivadas são dadas por

$$
s=\left[\begin{array}{l}
\mathrm{s}_{\eta} \\
\mathrm{s}_{\theta}
\end{array}\right]=\left[\begin{array}{l}
\partial \ln \lambda / \partial \eta \\
\partial \ln \lambda / \partial \theta
\end{array}\right]
$$

e

$$
H=\left[\begin{array}{ll}
H_{\eta \eta} & H_{\eta \theta} \\
H_{\theta \eta} & H_{\theta \theta}
\end{array}\right]=\left[\begin{array}{ll}
\partial^{2} \ln \lambda / \partial \eta^{\mathrm{t}} \partial \eta & \partial^{2} \ln \lambda / \partial \eta^{\mathrm{t}} \partial \theta \\
\partial^{2} \ln \lambda / \partial \theta^{\mathrm{t}} \partial \eta & \partial^{2} \ln \lambda / \partial \theta^{\mathrm{t}} \partial \theta
\end{array}\right]
$$

Os estimadores de máxima verossimilhança de $\eta$ e $\theta, \hat{\eta}$ e $\hat{\theta}$, correspondem às soluções de $\mathbf{s}=\mathbf{0}$, se elas estiverem contidas no espaço paramétrico definido pelo modelo (isto é, se $\Sigma_{i j}(\theta)$ for positiva definida para $i=1, \ldots, b$ e $\left.j=1, \ldots, t\right)$ e se $\mathbf{H}(\hat{\eta}, \hat{\theta})$ for negativa definida (para garantir que $\hat{\eta}$ e $\hat{\theta}$ são pontos de máximo).

Em geral, não há soluções explícitas e é necessário utilizar métodos iterativos. Jennrich e Schluchter (1986) descrevem os algoritmos Newton-Raphson, "Scoring" de Fisher e EM híbrido com "Scoring". Laird e Ware (1982) sugerem a aplicação do algoritmo EM através da formulação de modelos de efeitos aleatórios. Para maiores detalhes, inclusive aspectos computacionais relativos a esses processos iterativos, consultar Andreoni (1989).

Testes de hipóteses referentes a combinações lineares da forma $\mathrm{Ho}_{0} \mathbf{C} \eta \mathbf{=} \mathbf{0}$, onde C $(c \times(b-1+p(p+1) / 2))$ é uma matriz de constantes conhecidas de posto $c(c<b-1+$ $p(p+1) / 2)$, podem ser realizados através da estatística de Wald

$$
Q_{c}=(C \hat{\alpha})^{t}\left[C\left(\sum_{i=1}^{b} \sum_{j=1}^{t} W_{i j}^{\mathrm{t}} \hat{\Sigma}_{i j}^{-1} W_{i j}\right)^{-1} C^{\mathrm{t}}\right]^{-1}(C \hat{\alpha})
$$

Sob $H o, Q_{c}$ tem distribuição assintótica Qui-Quadrado com $c$ graus de liberdade. 


\section{Apêndice B}

\section{Comentários sobre o "software" utilizado neste capítulo:}

Existem muitos pacotes que podem ser utilizados na Análise de Medidas Repetidas. Alguns apresentam subrotinas especiais; em outros, esse tipo de análise é um caso particular de modelos mais gerais. Em outros, ainda, há a possibilidade de se desenvolverem programas para realizar a análise. Descrevemos brevemente aqui os recursos oferecidos pelos pacotes BMDP, SAS e SPSS, bem como os programas utilizados nas análises.

\section{B.1. BMDP / 3V}

Neste programa são utilizados métodos de máxima verossimilhança para executar análises de (co)variância com efeitos fixos e aleatórios.

\section{B.2. BMDP / 4V}

Este módulo executa análises de (co)variância univariada e multivariada incluindo medidas repetidas. São fornecidos resultados uni e multivariados. 
Para explicações mais detalhadas sobre alguns comandos utilizados no módulo 4V, ver Davidson e Toporek (1983).

\section{B.3. BMDP / 5V}

Este programa utiliza os métodos de Máxima Verossimilhança ou Máxima Verossimilhança Restrita para a obtenção de estimadores e testes de hipóteses para modelos com matrizes de covariância com diferentes estruturas, incluindo simetria composta, auto-regressiva, não estruturada e modelos de efeitos aleatórios).

Para as estimativas de máxima verossimilhança, podem-se usar os algoritmos de Newton-Raphson, scoring de Fisher e EM, e para as estimativas de máxima verossimilhança restrita, os métodos disponíveis são o algoritmo EM generalizado e um algoritmo quasi-scoring.

\section{B.4. BMDP / 8V}

Este programa pode ser utilizado para a análise de modelos mistos provenientes de vários tipos de planejamento (desde que sejam completos) e permite a presença tanto de efeitos fixos como de aleatórios. Permite que contrastes sejam especificados e executa análises multivariadas.

\section{B.5. SAS GLM e SPSS/Manova}

Ambos os programas ajustam modelos lineares gerais utilizando a técnica de mínimos quadrados, executando análises uni e multivariada.

Os testes univariados são apresentados com as correções de Greenhouse-Geisser e de Huynh-Feldt. Testes de esfericidade podem ser requisitados e as estatísticas multivariadas também são fornecidas.

Apresentamos a seguir alguns dos programas para BMDP utilizados neste trabalho. Os dados analisados são referentes ao Exemplo 1.1 e encontram-se em um arquivo ASCII de nome EXEMP11.DAT. 
PROGRAMA PARA BMDP/AV - MODELO COM INTERAÇÃO BLOCOS $\times$ TEMPO

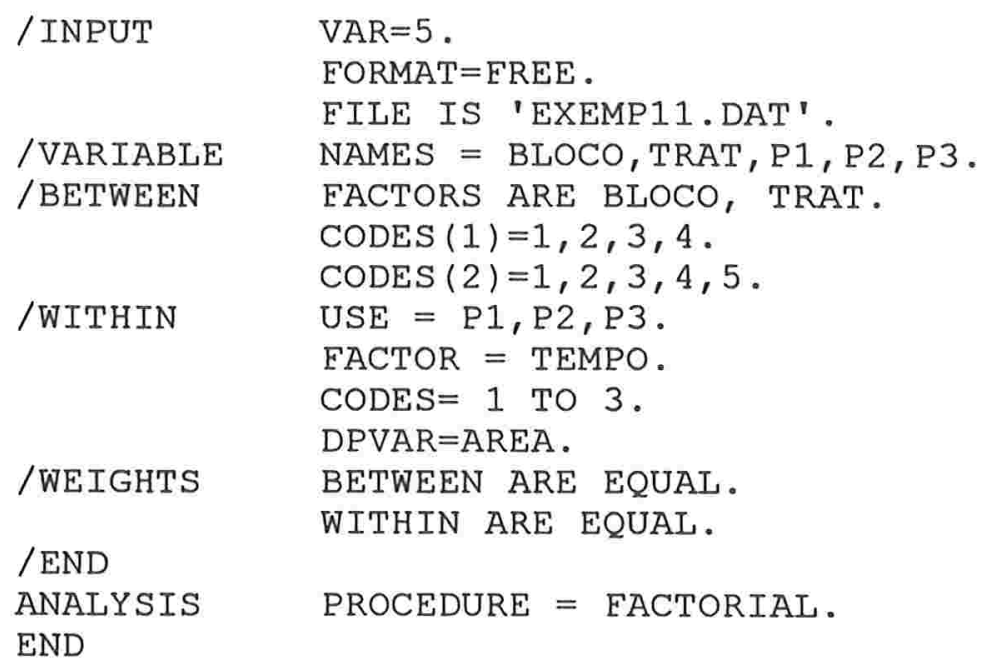

/END

ANALYSIS PROCEDURE = STRUC.

END BFORM = 'TRAT'+ BLOCO./ 
PROGRAMA PARA BMDP/AV - MODELO SEM INTERAÇÃO BLOCOS $\times$ TEMPO

(FINALIDADE: TESTAR A HIPÓTESE DE INEXISTÊNCIA DE EFEITO DE TRATAMENTO E OBTER AS ESTIMATIVAS DOS EFEITOS DE BLOCOS PARA O CÁLCULO DOS RESÍDUOS).

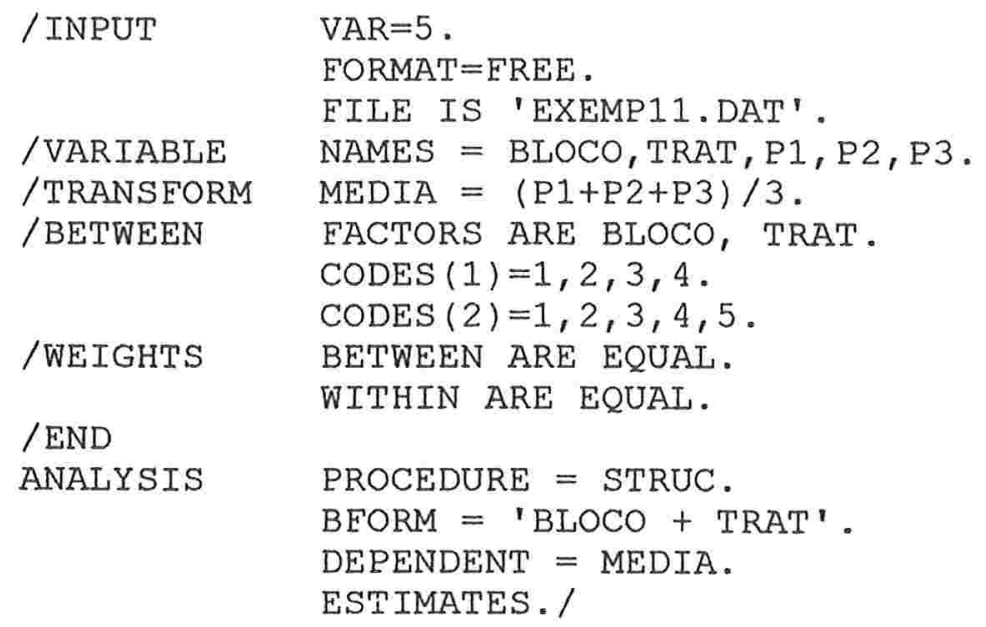

/END

ANALYSIS PROCEDURE $=$ STRUC. ESTIMATES./

END

PROGRAMA PARA BMDP/4V - ANÁLISE DE RESÍDUOS E TESTE DA HIPÓTESE H1 (VER PAG. 43)

OBS: O ARQUIVO RESID11.DAT CONTÉM OS RESÍDUOS DOS DADOS DO EXEMPLO 1.1 (DADOS ORIGINAIS - EFEITO DE BLOCOS)

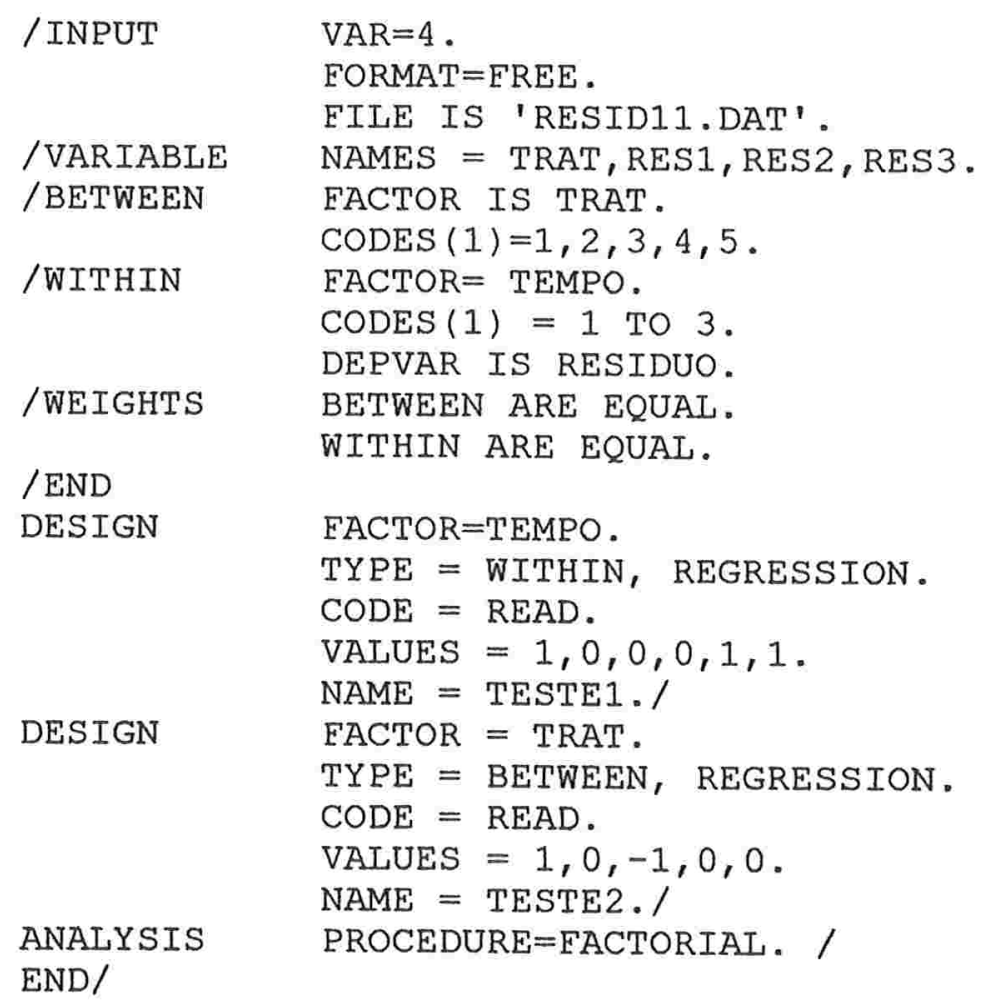


PROGRAMA PARA BMDP/5V - MODELO COM INTERAÇÃO BLOCOS $\times$ TEMPO SIGMA COM PADRÃO UNIFORME

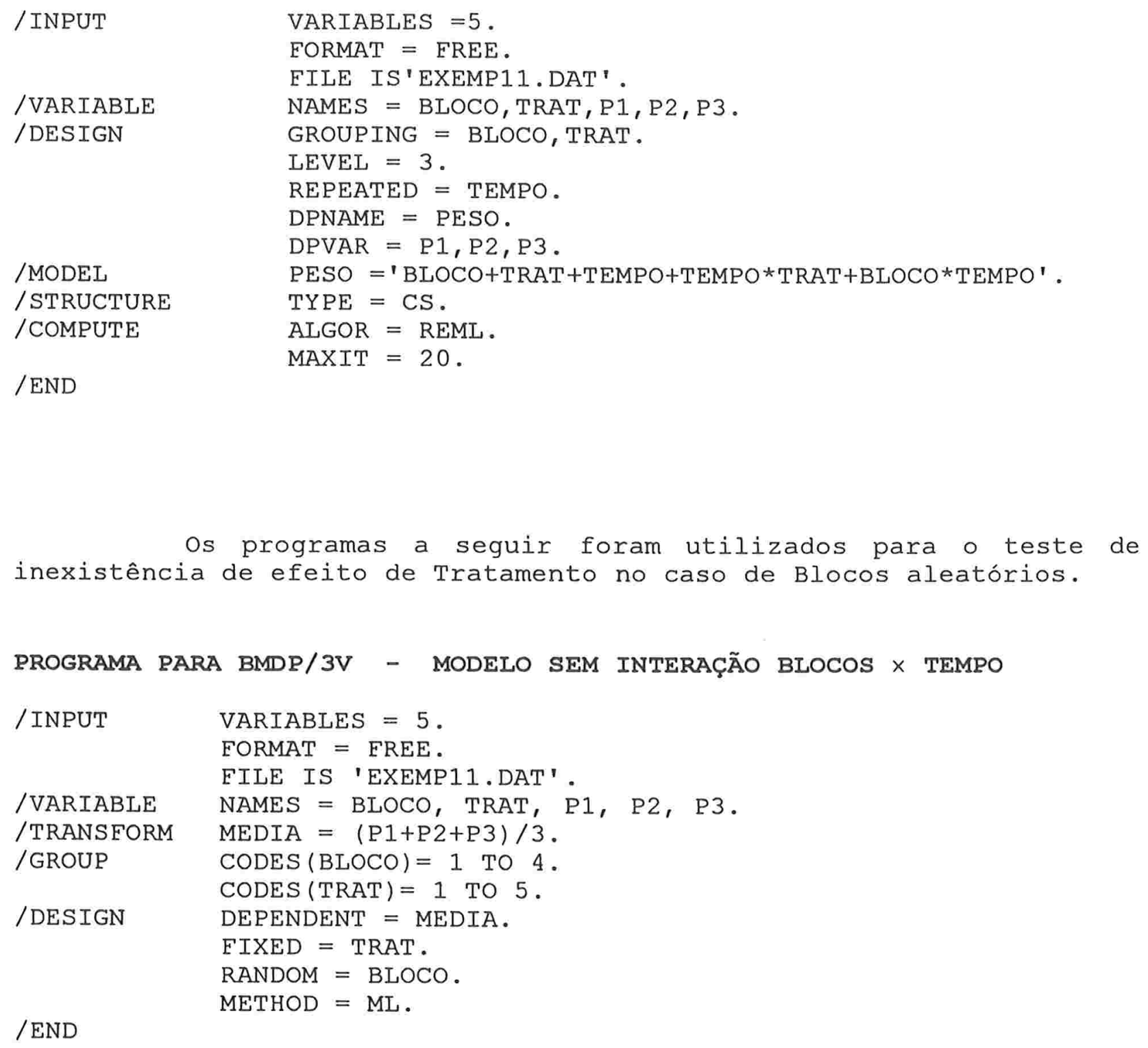

/END

Os programas a seguir foram utilizados para o teste de inexistência de efeito de Tratamento no caso de Blocos aleatórios. 
PROGRAMA PARA BMDP/8V - MODELO SEM INTERAÇÃO BLOCO $\times$ TEMPO

(FINALIDADE: TESTAR A HIPÓTESE DE INEXISTÊNCIA DE EFEITO DE TRATAMENTO)

$\begin{array}{ll}/ \text { INPUT } & \text { VAR }=5 . \\ & \text { FORMAT }=\text { FREE. } \\ & \text { FILE IS 'EXEMP11. DAT' } \\ \text { /VARIABLE } & \text { NAMES }=X 1, \mathrm{X} 2, \mathrm{P} 1, \mathrm{P} 2, \mathrm{P} 3 . \\ \text { /TRANSFORM } & \text { MEDIA }=(\mathrm{P} 1+\mathrm{P} 2+\mathrm{P} 3) / 3 . \\ \text { /DESIGN } & \text { LEVELS ARE } 4,5 . \\ & \text { NAMES }=\text { BLOCO, TRAT. } \\ & \text { DEPEND }=\text { MEDIA. } \\ & \text { FIXED }=\text { TRAT. } \\ & \text { RANDOM }=\mathrm{BLOCO} . \\ & \text { MODEL }=' \mathrm{~T}, \mathrm{~B} ' .\end{array}$




\section{Referências Bibliográficas}

Albohali, M. N. (1983). A time series approach to the analysis of repeated measures designs . Ph.D. dissertation, Kansas State University.

Andrade, D. F. e Singer, J. M. (1986). Análise de Dados Longitudinais. VII SINAPE (Campinas). São Paulo: Associação Brasileira de Estatística.

Andrade, D. F., e Moura, M. F., Ternes, S. (1988). Uso do SOC na análise de modelos lineares multivariados. Relatório Técnico. NTIA/EMBRAPA.

Andrade, D. F. e Singer, J. M. (1994). Profile Analysis for Randomized Complete Block Experiments. Relatório Técnico No. 6772, São Paulo: IME - Universidade de São Paulo.

Andreoni, S. (1989). Modelos de Efeitos Aleatórios para Análise de Dados Longitudinais Não-Balanceados em Relação ao Tempo. Dissertação de Mestrado, São Paulo: IME Universidade de São Paulo.

Box, G. E. P. (1954). Some theorems on quadratic forms applied to the study of analysis of variance problems. The Annals of Mathematical Statistics 25, 290-302, 484-498.

Chi, E. M. and Reinsel, G. C. (1989). Models for longitudinal data with random effects and AR(1) errors. Journal of the American Statistical Association 84, 452-459.

Cook, N. R. and Ware, J. W. (1983). Design and analysis methods for longitudinal research. Annual Review of Public Health 4, 1-23.

Davidson, M. and Toporek, J. (1983). BMDP Technical Report, 67. (BMDP4V General Univariate and Multivariate Analysis of Variance).

Dielman, T. E. (1983). Pooled cross sectional and time series data: a survey of current statistical methodology. The American Statistician 37, 112-122.

Domenech, C. H. (1989). Métodos Exploratórios e Uso de Medidas Resumo para Análise de Dados Longitudinais. Dissertação de Mestrado, São Paulo: IME Universidade de São Paulo.

Gabriel, K. R. (1961). The model of ante-dependence for data of biological growth. Bulletin Institut International Statistique (Paris) 39, 253-264. 
Gabriel, K. R. (1962). Ante-dependence analysis of an ordered set of variables. The Annals of Mathematical Statistics 33, 201-212.

Geisser, S. and Greenhouse, S. W. (1958). An extension of Box's results on the use of the F distribution in multivariate analysis. The Annals of Mathematical Statistics 29, 855-891.

Goldstein, H. (1979). The Design and Analysis of Longitudinal Studies. London: Academic Press.

Grande, R. H. M., Singer, J. M., Santos, J. F. F. and Nicolau, J. (1992). Effectiveness of three mouthrinses to inhibit acid formation by dental plaque under home-use conditions. Clinical Preventive Dentistry 14, 19-23.

Greenhouse, S. W. and Geisser, S. (1959). On Methods in the Analysis of Profile Data. Psycometrika 24, 95-112.

Harville, D. A. (1977). Maximum likelihood approaches to variance component esimation and to related problems. Journal of the American Statistical Association 72, 320-340.

Heckman, J. J. and Singer, B. (1985). Longitudinal Analysis of Labour Market Data. Cambridge: University Press.

Hoffman, D. J., Franson, O. H., Patee, C. M., Bunck, C. M. and Anderson, A. (1985). Survival, growth and accumulation of ingested lead in nestling American Kestrels (Falco spaverius). Archive of Environmental Contamination and Toxicology, 14 8994.

Huynh, H. and Feldt, L. S. (1970). Condition under which mean square ratios in repeated measurements designs have exact F-distributions. Journal of the American Statistical Association 65, 1582-1589.

Huynh, H. and Feldt, L. S. (1976). Estimation of the Box correction for degrees of freedom from sample data in randomized block and split-plot designs. Journal of Educational Statistics 1, 69-82.

Jennrich, R. I. and Schluchter, M. D. (1986). Unbalanced repeated measures models with structured covariance matrices. Biometrics 42, 805-820.

Kenward, M. G. (1987). A method for comparing profiles of repeated measurements. Applied Statistics 36, 296-308. 
Kowalski, C. J. and K. E. Guire (1974). Longitudinal data analysis. Growth 38, 131169.

Laird, N., Lange, N. and Stram, D. (1987). Maximum likelihood computations with repeated measures: application of the EM algorithm. Journal of the American Statistical Association 82, 97-105.

Laird, N. M. and Ware, J. H. (1982). Random-effects models for longitudinal data. Biometrics 47, 963-974.

Lavange, L. M. and Helms, R. W. (1983). The analysis of incomplete longitudinal data with time series covariance structure. Artigo apresentado no Joint Statistical Meetings, Toronto, Canadá.

Lindstrom, M. J. and Bates, D. M. (1988). Newton-Raphson and EM algorithms for linear mixed-effects models for repeated measures data. Journal of the American Statistical Association 83, 1014-1022.

Mauchly, J. M. (1940). Significance test for sphericity of normal $n$-variate distribution. Annals of Mathematical Statistics 11, 204-209.

Milliken, G. A. and Johnson, D. E. (1984). Analysis of Messy Data, v.1: Designed Experiments. Belmont, California: Wadsworth, Inc.

Morrison, D. F. (1976). Multivariate Statistical Methods, $2^{\text {nd }}$ edition. New York: McGraw Hill.

Nesselroade, J. R. and Baltes, P. B. (1979). Longitudinal Research in the Study of Behaviour and Development. New York: Academic Press.

Pantula, S. G. and Pollock, L. H. (1985). Nested analysis of variance with autocorrelated errors. Biometrics 41, 909-920.

Papescu, S. (1995). Análise de Perfis com Dados Incompletos. Dissertação de Mestrado, São Paulo: IME - Universidade de São Paulo.

Rouanet, H. and Lépine, D. (1970). Comparison between treatments in a repeatedmeasurement design: ANOVA and multivariate methods. Brittish Journal of Mathematical Statistics, 23, 147-163.

Rowell, J. G. and Walters, D. E. (1976). Analyzing data with repeated observations on each experimental unit. Journal of Agricultural Science 87, 423-432. 
Roy, S. N. (1953). On a heuristic method of test construction and its use in multivariate analysis. The Annals of Mathematical Statistics, 24, 220-238.

Samuels, M.L., Casella, G. and McCabe, G.P. (1991). Interpreting blocks and random effects (with comments by T. P. Speed, R. R. Hocking, D. A. Marville and R. H. Bremer). Journal of the American Statistical Association $\quad 86,798-821$

Schaalje, B., Zhang, J., Pantula, S. G. and Pollock, K. H. (1987). REMAC: Repeated Measures analysis for complete data. North Carolina State University. Institute of Statistics Mimeo Series No. 1911.

Schaalje, B., Zhang, J., Pantula, S. G. and Pollock, K. H. (1991). Analysis of repeated measurements data from randomized block experiments. Biometrics 47, 813-824.

Seber, G. A. F. (1984). Multivariate Observations. New York: John Wiley \& Sons.

Singer, J. M. (1977). Análise de Curvas de Crescimento . Dissertação de Mestrado, São Paulo: IME - Universidade de São Paulo.

Singer, J. M. and Domenech, C. H. (1989). Medidas Resumo: Uma alternativa para Análise de Dados Longitudinais. Anais do 3o SEAGRO - Lavras, M. G.: Escola Superior de Agricultura de Lavras.

Steel, R. G. D. and Torrie, J. H. (1980). Principles and procedures of Statistics, 2nd ed. New York: McGraw-Hill.

Thompson, R. (1969). Iterative estimation of variance components for non-orthogonal data. Biometrics 25, 767-773.

Timm (1975). Multivariate Analysis with Applications in Education and Psychology. Monterey, California: Brooks-Cole.

Timm (1980). Multivariate analysis of variance of repeated measurements. In: Handbook of Statistics (vol. 1). P.R. Krishnaiah (ed.). North-Holland, Amsterdam, 41-87.

Wallestein, S. and Fleiss, J. L. (1979). Repeated measurements analysis of variance when the correlations have a certain pattern. Psychometrika 44, 229-233.

Ware, J. H. (1985). Linear models for the analysis of longitudinal studies. The American Statistician 39, 95-101.

Wilks, S.S. (1932). Certain generalizations in the analysis of variance. Biometrika 24, 471-494. 
Winer, B. J. (1971). Statistical principles in experimental design. $2^{\text {nd }}$ ed. New York: McGraw-Hill.

Wishart, J. (1938). Growth rate determination in nutrition studies with the bacon pig, and their analysis. Biometrika 30, 18-28.

Zhang, J., Schaaje, B., Pantula, S. G., and Pollock, K. H. (1987). REMACRB: Repeated Measures Analysis for Complete data from Randomized Block experiments. Technical ' Report. Institute of Statistics - North Carolina State University. 